\title{
Preparing the Periphery for a Subsequent Behavior: Motor Neuronal Activity during Biting Generates Little Force but Prepares a Retractor Muscle to Generate Larger Forces during Swallowing in Aplysia
}

\author{
Hui Lu, ${ }^{1}$ Jeffrey M. McManus, ${ }^{1}$ Miranda J. Cullins, ${ }^{1}$ and Hillel J. Chiel ${ }^{1,2,3}$ \\ ${ }^{1}$ Department of Biology, ${ }^{2}$ Department of Neurosciences, and ${ }^{3}$ Department of Biomedical Engineering, Case Western Reserve University, Cleveland, Ohio \\ 44106-7080
}

\begin{abstract}
Some behaviors occur in obligatory sequence, such as reaching before grasping an object. Can the earlier behavior serve to prepare the musculature for the later behavior? If it does, what is the underlying neural mechanism of the preparation? To address this question, we examined two feeding behaviors in the marine mollusk Aplysia californica, one of which must precede the second: biting and swallowing. Biting is an attempt to grasp food. When that attempt is successful, the animal immediately switches to swallowing to ingest food. The main muscle responsible for pulling food into the buccal cavity during swallowing is the I3 muscle, whose motor neurons B6, B9, and B3 have been previously identified. By performing recordings from these neurons in vivo in intact, behaving animals or in vitro in a suspended buccal mass preparation, we demonstrated that the frequencies and durations of these motor neurons increased from biting to swallowing. Using the physiological patterns of activation to drive these neurons intracellularly, we further demonstrated that activating them using biting-like frequencies and durations, either alone or in combination, generated little or no force in the I3 muscle. When biting-like patterns preceded swallowing-like patterns, however, the forces during the subsequent swallowing-like patterns were significantly enhanced. Sequences of swallowing-like patterns, either with these neurons alone or in combination, further enhanced forces in the I3 muscle. These results suggest a novel mechanism for enhancing force production in a muscle, and may be relevant to understanding motor control in vertebrates.
\end{abstract}

Key words: Aplysia; behavioral sequence; feeding; motor neuron; multifunctionality; muscle

\section{Introduction}

Could a behavior that is an obligatory precursor of another behavior prepare the musculature for that subsequent behavior? Bernstein (1967, pp 110-111) hypothesized that the periphery should be properly prepared for "the right impulse at the right moment." For example, anticipatory postural movements in humans significantly affect subsequent postural movements when subjects attempt to stop heavy moving objects (Santos et al., 2010a, b). In smooth muscle, prior stretch can affect the amount of force that a muscle can generate, and modulatory compounds can also significantly alter force output (Halayko and Solway, 2001; Burnstock, 2013). To determine the behavioral relevance of preparatory activity from one behavior to the next, in vivo activity

\footnotetext{
Received Feb. 12, 2014; revised Feb. 9, 2015; accepted Feb. 15, 2015.

Author contributions: H.L., J.M.M., M.J.C., and H.J.C. designed research; H.L., J.M.M., and M.J.C. performed research; H.L., J.M.M., M.J.C., and H.J.C. analyzed data; H.L., J.M.M., and H.J.C. wrote the paper.

This work was supported by National Institutes of Health Grant NS047073 and National Science Foundation Grants DMS-1010434 and IIS-1065489.

The authors declare no competing financial interests.

Correspondence should be addressed to Hillel J. Chiel, PhD, Department of Biology, Case Western Reserve University, 2080 Adelbert Road, Cleveland, 0H 44106-7080. E-mail: hj@@case.edu.

DOI:10.1523/JNEUROSCI.0614-14.2015

Copyright $\odot 2015$ the authors $\quad 0270-6474 / 15 / 355051-16 \$ 15.00 / 0$
}

of motor neurons should be monitored as an animal transitions from a precursor behavior to the subsequent behavior.

To address this question, we studied feeding in Aplysia californica, a robust and tractable system allowing analyses of motor neuronal control, biomechanics, and neuromodulation at the level of individual identified motor neurons. Previous studies in Aplysia have demonstrated that one phase of the behavior can prepare the system for a subsequent phase of the same behavior biomechanically (Sutton et al., 2004; Ye et al., 2006a). In addition, muscle properties can be altered in this system to enhance its ability to generate forces through neuromodulation (Weiss et al., 1978, 1992; Fox and Lloyd, 1998, 2000).

In Aplysia, one behavior (biting) is an obligatory precursor for a second behavior (swallowing). A bite is an attempt to grasp food; only when this attempt is successful does the animal switch to swallowing (Kupfermann, 1974). During swallowing, animals must tightly grasp food and strongly pull it into the buccal cavity (i.e., strongly retract the grasper; Neustadter et al., 2007). The muscle mediating the retraction phase is known as I3 (Howells, 1942). The motor neurons that innervate the muscle are also known (Church and Lloyd, 1991, 1994), and previous studies have shown that prior activity of some of these motor neurons (B3, B9, and B38) can increase the contraction amplitude and 
A

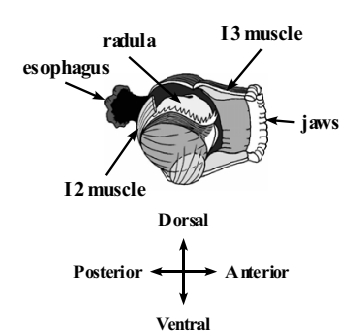

B

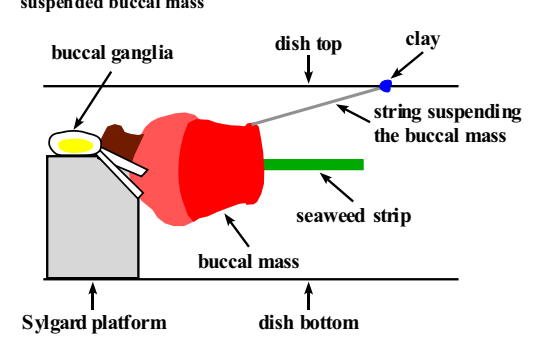

C anchored buccal mass

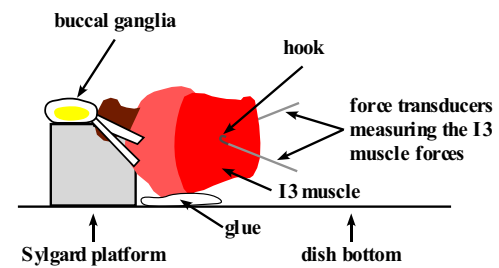

Figure 1. Schematics of the Aplysia buccal mass and two in vitro preparations: suspended and anchored buccal mass preparations. $A$, Schematic cut-through drawing of a side view of the Aplysia buccal mass, modified from Figure 1 in Sutton et al. (2004) and Figure 1 in McManus et al. (2014). The radula is the pincers-like food grasper, whose two halves close together to grasp food. The 12 muscle is a thin, sheet-like muscle that wraps around the back of the buccal mass, which contracts to push the grasper toward the jaws (protraction). The I3 muscle is a ring-shaped muscle at the front of the buccal mass, which contracts to push the grasper back toward the esophagus (retraction). B, Suspended buccal mass preparation (for full details, see McManus et al., 2012). This in vitro preparation makes it possible to record from individual motor neurons or stimulate them in the buccal ganglion while observing biting or swallowing responses in the feeding apparatus (the buccal mass). C, Anchored buccal mass preparation (for full details, see Lu et al., 2013). This in vitro preparation makes it possible to record from individual motor neurons or stimulate them in the buccal ganglion while measuring forces generated in the 13 muscle.

relaxation rate of $\mathrm{I} 3$ during later activation of the same motor neurons (Fox and Lloyd, 1997, 2001; Keating and Lloyd, 1999).

We hypothesize that the primary function of the key I3 motor neurons in biting is not to generate force for retraction, but to prepare I3 for force generation in swallowing, so that once Aplysia grasps food, it can generate a large retraction force in the initial swallow, allowing an animal to successfully ingest food. In this study, we report that (1) B6, B9, and B3 are the key motor neurons for I3 force generation during the retraction phase of swallowing; (2) the in vivo firing of B6, B9, and B3 generates little or no force during biting, but prepares I 3 for force generation in the initial swallow; and (3) the repeated firing and combined activity of B6, B9, and B3 in swallowing strongly enhances I3 forces for subsequent swallows.

\section{Materials and Methods}

Animals. A. californica weighing 200-450 g (Marinus Scientific) were maintained in an aerated aquarium containing artificial seawater (Instant Ocean, Aquarium Systems) kept at $16 \pm 1{ }^{\circ} \mathrm{C}$. Animals were fed every other day with dried seaweed (nori). Before experiments, animals were presented with seaweed to measure the intervals between bites. Animals that met the standard criteria for healthy behavior (i.e., displayed strong bites at 3-5 s intervals; Kupfermann, 1974) were selected for use (350-450 $\mathrm{g}$ for in vivo experiments; 200-350 $\mathrm{g}$ for in vitro experiments).

Electrodes. Intracellular glass electrodes were pulled from singlebarreled capillary glass (catalog \#6150, AM Systems) using a Flaming/ Brown micropipette puller (model P-80/PC, Sutter Instruments). Their inner diameters were $3-6 \mu \mathrm{m}$. Electrodes were backfilled with $3 \mathrm{~m}$ potassium acetate before use. The bridge was balanced for stimulation. Intracellular signals were amplified using a DC-coupled amplifier (model 1600, A-M Systems).

Extracellular glass electrodes were also pulled from single-barreled capillary glass (catalog \#6150, A-M Systems), using a Flaming/Brown micropipette puller (model P-80/PC, Sutter Instruments). Their inner diameters were $\sim 40 \mu \mathrm{m}$. Electrodes were backfilled with Aplysia saline before use. Currents were supplied by a stimulus isolator (model A-360, WPI).

Extracellular suction electrodes were made from polyethylene tubing (catalog \#427421, Becton Dickinson; outer diameter, $1.27 \mathrm{~mm}$; inner diameter, $0.86 \mathrm{~mm}$ ) as described in Lu et al. (2013). They were also backfilled with Aplysia saline before use.

Hook electrodes were made from enamel-coated stainless steel wire (catalog \#100194, California Fine Wire; 0.001 inch diameter, $\sim 2$ feet long) as described in Cullins and Chiel (2010).

All extracellular recording signals (i.e., from extracellular soma electrodes, extracellular suction electrodes, and hook electrodes) were amplified using an AC-coupled differential amplifier (model 1700, A-M
Systems). In vivo nerve recordings were filtered using a $300 \mathrm{~Hz}$ high-pass filter and a $1 \mathrm{kHz}$ low-pass filter. In vivo muscle recordings were filtered using a $10 \mathrm{~Hz}$ high-pass filter and a $1 \mathrm{kHz}$ low-pass filter. In vitro nerve and soma recordings were filtered using a $300 \mathrm{~Hz}$ high-pass filter and a $500 \mathrm{~Hz}$ low-pass filter. In vitro muscle recordings were filtered using a 10 $\mathrm{Hz}$ high-pass filter and a $500 \mathrm{~Hz}$ low-pass filter.

Experimental preparations. An in vivo preparation and two in vitro preparations (i.e., a suspended buccal mass preparation and an anchored buccal mass preparation) were used in this study. The in vivo preparation was used to record neural and muscular activities as animals performed feeding behaviors. The suspended buccal mass preparation was used to monitor neural activity of individual motor neurons during feeding responses. The anchored buccal mass preparation was used to measure the muscle forces in I3 evoked by motor neuron stimulations or feeding-like motor patterns.

In vivo preparation. To record neural and muscular activities as animals perform feeding behaviors, hook electrodes were implanted onto a key muscle and nerves as described by Cullins and Chiel (2010). Briefly, animals weighing 350-450 g were selected and anesthetized by an injection of isotonic $\mathrm{MgCl}_{2}$ (333 mM). The volume (in milliliters) of the $\mathrm{MgCl}_{2}$ solution was equal to $30 \%$ of the animal's body weight (in grams). Hook electrodes were implanted on the I2 muscle that initiates the protraction phase of feeding (Fig. 1A; Hurwitz et al., 1996), the radular nerve $(\mathrm{RN})$ that carries motor neurons (B8a, B8b) mediating the closure of the food grasper (radula; Fig. 1A; Morton and Chiel, 1993a), and the buccal nerves 2 and 3 (BN2 and BN3) that carry motor neurons innervating the I3 muscle, which mediates the retraction phase of feeding (Church and Lloyd, 1991; Morton and Chiel, 1993a; Warman and Chiel, 1995; Fig. $1 A)$. After animals recovered from surgery, recordings from the I2 muscle, RN, BN2, and BN3 were obtained while animals performed feeding behaviors (biting and swallowing). A video camera was simultaneously used to record feeding behaviors.

In vitro suspended buccal mass preparation. To determine the neural activity patterns of individual motor neurons during feeding behaviors, an in vitro suspended buccal mass preparation was used to elicit biting and swallowing responses while extracellular soma electrodes were used to record from key motor neurons (Fig. 1B; McManus et al., 2012; Lu et al., 2013).

The protocol has been described in detail by McManus et al. (2012). Briefly, animals weighing 200-350 g were selected and anesthetized with an injection of $50 \%$ body weight isotonic $\mathrm{MgCl}_{2}(333 \mathrm{~mm})$. The buccal mass was dissected out with the buccal and cerebral ganglia attached. Hook electrodes were applied to the I2 muscle, RN, BN2, and BN3 for motor program measurements as was done in vivo by Cullins and Chiel (2010). In most in vitro experiments, BN2s and BN3s on both sides of the buccal ganglia were recorded to help identify motor neurons with ipsilateral and contralateral projections. 
The buccal mass and the buccal and cerebral ganglia were placed in a custom-made dish that could isolate the cerebral ganglion from the buccal mass and ganglia (McManus et al., 2012, their Fig. 1), so that the cholinergic agonist carbachol (10 mм in Aplysia saline) could be applied to the cerebral ganglion separately to elicit ingestion responses (Susswein et al., 1996). After pinning out the buccal and cerebral ganglia, the sheaths of the buccal ganglia were thinned, but not completely removed to allow better visualization and access to neurons' somata with extracellular glass electrodes. To suspend the buccal mass, a silk suture was threaded through the tissue anterodorsal to the jaws. The suture was then tightened and attached to two balls of modeling clay placed on the side of the dish (Fig. 1B).

To extracellularly record from a motor neuron, an extracellular glass electrode was gently pressed onto the sheath above the neuron's soma (Lu et al., 2008; McManus et al., 2012, their Fig. 5). A short anodic current pulse (e.g., $6 \mathrm{~ms}, 200 \mu \mathrm{A}$ ) was applied through the extracellular glass electrode to activate the neuron (Lu et al., 2008). Once the neuron fired, the extracellular soma channel was then quickly switched from stimulation to recording mode. Observations of one-for-one spikes on the extracellular soma channel and the corresponding nerve channel(s) were used to help confirm identities of motor neurons (McManus et al., 2012; Lu et al., 2013). The extracellular glass electrode was left in place to monitor the neuron's activity during feeding responses. Simplified criteria for extracellularly identifying different motor neurons were established by Lu et al. (2013). Specifically, B6 and B9 are the only two motor neurons that project through the $\mathrm{BN} 2 \mathrm{~s}$ and $\mathrm{BN} 3 \mathrm{~s}$ bilaterally, and $\mathrm{B} 6$ is medial to B9 (Church et al., 1991, their Fig. 2). B3 only projects through the ipsilateral BN2, and its BN2 unit is always the largest. All of these are motor neurons for the I3 muscle (Church et al., 1991).

After neurons were located, biting responses were induced by applying the cholinergic agonist carbachol to the cerebral ganglion in the isolated chamber so that it would not diffuse into other chambers that contain the buccal ganglia and buccal muscles (Susswein et al., 1996). The details of carbachol application have also been described in McManus et al. (2012) and Lu et al. (2013). Swallowing responses were obtained by placing a strip of seaweed ( $10 \mathrm{~cm}$ long, $0.25 \mathrm{~cm}$ wide) in the feeding grasper during a bite; this induced the preparation to swallow the seaweed strip (Fig. $1 B$ ).

In vitro anchored buccal mass preparation. Since the suspended buccal mass was not stationary during feeding responses, it was not feasible to precisely measure muscle forces in that preparation. Thus, we developed an in vitro anchored buccal mass preparation (Fig. 1C; Lu et al., 2013). Because the I3 muscle is similar to vertebrate smooth muscle (Gallo et al., 2004), it does not generate twitch responses to presynaptic action potentials; rather, it generates excitatory junction potentials (EJPs) that summate to cause muscle contraction (Cohen et al., 1978).

Animals weighing 200-350 g were selected and anesthetized and the buccal mass was dissected out as described above. The I3 muscle, which forms a ring around the cartilage of the jaws, and serves as the main retractor muscle, is found beneath the superficial, very thin muscle I1 (Howells, 1942), which tightly adheres to I3, and thus cannot be dissected away without causing damage to I3. Force measurements, however, primarily represent contractions of I3, because we are activating I3's motor neurons (B6, B9, and B3; Church et al., 1991). Thus, throughout the rest of the paper, we will refer solely to the I3 muscle, even though force measurements were done of the I1/I3 complex. Since I1/I3 is primarily innervated by BN2 (Scott et al., 1991, they referred to BN2 as nerve 5), all nerves except BN2s were severed from the buccal mass, so that only I1/I3 would be innervated during the experiments, and thus would be the sole source of force. The I1/I3 lumen was left intact, so that the muscle would be in a configuration similar to its physiological state. Hook electrodes were attached to BN2s and BN3s bilaterally for motor program measurements and motor neuron identification.

The buccal mass and the buccal and cerebral ganglia were then placed in a custom-made dish specialized for the in vitro anchored buccal mass preparation (Lu et al., 2013, their Fig. 1). To stabilize the buccal mass, it was glued to the bottom of the dish on its ventral surface (Fig. 1C). After the buccal and cerebral ganglia were pinned to Sylgard, the sheaths of the buccal ganglia were either thinned or removed to allow extracellular or intracellular glass electrodes to access neurons' somata, respectively. Two extracellular suction electrodes were attached to the I2 nerve and $\mathrm{RN}$ for motor program measurement (Lu et al., 2013).

Force transducers (Grass Technologies) were then attached to the center of the lateral regions of the I1/I3 complex using hooks and silk sutures, enabling us to measure the muscle forces evoked by motor neuron stimulations or generated during feeding-like motor patterns.

Characterizing the BN2 motor program patterns during the retraction phase. As described by Morton and Chiel (1993a, b) and Lum et al. (2005), the BN2 recordings almost always contained three classes of spikes, based on their spike amplitude, labeled as "small," "medium," and "large." To identify the motor neurons corresponding to these different BN2 units and to determine their neural activity patterns during feeding, we used extracellular soma recordings to record from individual motor neurons during feeding responses in the suspended buccal mass preparation (McManus et al., 2012).

Motor neuron identification. The protraction phase of a feeding response was defined by the I 2 muscle activity (Fig. $2 A$, white bar). The retraction phase was defined by a BN2 burst that starts at or after the end of the I2 activity (Fig. 2A, black bar). We observed that the large BN2 units fired in the middle retraction phase. The medium BN2 units fired in the middle retraction phase as well as during the interpattern interval and the protraction phase. The small BN2 units fired throughout the entire retraction phase. There were some even smaller BN2 units firing within or out of the retraction phase. To distinguish the small BN2 units from these very small units, we labeled large, medium, and small BN2 units as the "largest," "second largest," and "third largest" groups of BN2 units.

An amplitude window discriminator algorithm was used to detect BN2 units of different spike sizes (Morton and Chiel, 1993a). The oneto-one relationships between the extracellular soma and the BN2 recordings show that $\mathrm{B} 6$ and $\mathrm{B} 9$ corresponded to the second largest group of BN2 units (Fig. 2B1,B2, yellow dashed lines) and B3 corresponded to the largest group of BN2 units (Fig. 2B3, red dashed lines). The latency from the B6 soma spikes to the corresponding BN2 spikes was $9.9 \pm 0.6 \mathrm{~ms}$ (mean $\pm \mathrm{SD}$, from 14 pairs of spikes in one experiment). The latency from the B9 soma spikes to the corresponding BN2 spikes was $11.3 \pm 0.4$ $\mathrm{ms}$ (mean $\pm \mathrm{SD}$, from 14 pairs of spikes in one experiment). The latency from the $\mathrm{B} 3$ soma spikes to the corresponding BN2 spikes was $7.6 \pm 0.1$ $\mathrm{ms}$ (mean $\pm \mathrm{SD}$, from 10 pairs of spikes in one experiment). Note that the spike sizes of soma recordings were sometimes variable in the suspended buccal mass preparation. The extracellular recording signals were affected by the distance between the target soma and the extracellular electrode (Lu et al., 2013). As the buccal mass performed behavioral responses, the buccal ganglia moved due to contractions of the buccal muscles that were attached to the nerves of the buccal ganglia. Thus, the distances between the target soma and the extracellular electrode would sometimes cause small variations of the soma signals.

B6 and B9 fired in the middle retraction phase. Without extracellular soma recordings, it was difficult to distinguish B6 neurons from B9 neurons. Note that there is one $\mathrm{B} 6$ and one $\mathrm{B} 9$ in each buccal hemiganglion, and each of these neurons usually projects through both ipsilateral and contralateral BN2s. Thus, the second largest group of units on a single BN2 typically corresponded to at least one of the four B6/B9 neurons, but the other B6/B9 neurons might produce smaller units. These medium units were designated as block 2 (Fig. $2 A$, yellow box).

B3 usually started firing after B6/B9 started and ended slightly before or as B6/B9 ended. However, occasionally, B3 started firing early, even before B6/B9 started (our unpublished results). Since B3 units are always the largest on $\mathrm{BN} 2$, it is easy to detect them directly from $\mathrm{BN} 2$ recordings. These large units were designated as block 3 (Fig. 2A, red box).

Morton and Chiel (1993b) have shown that B10 corresponded to the third largest group of BN2 units, and that it started firing at the onset of the $\mathrm{BN} 2$ burst and continued firing into the middle retraction phase overlapping the activity of B6, B9, and B3. Based on our unpublished results, $\mathrm{B} 39$ and several other unidentified motor neurons might also fire in the early phase of retraction (Fig. 2A, green box). Because of the limitations of the extracellular identification technique, we were not able to reliably identify these neurons and characterize their neural activity 
patterns in the suspended buccal mass preparation (Lu et al., 2013). It was also difficult to identify these units directly from the BN2 recordings without soma recordings in vivo or in vitro. These early small units were designated as block 1 (Fig. 2A, green box).

Lu et al. (2013) have shown that B43 also corresponded to the third largest group of BN2 units and it usually bursts at the end of the retraction phase at high frequency (Fig. $2 A$, blue box). This made it easy to detect B43 bursting units directly from $\mathrm{BN} 2$ recordings without extracellular soma recordings. However, in swallowing, B43 was more active and usually started much earlier, overlapping the activity of B6, B9, and B3 as well as other small BN2 units, which made it difficult to identify the early B43 units (our unpublished results). These late small units were designated as block 4 (Fig. 2A, blue box).

In Figure $2 A$, we noticed that in addition to B6 and B9, there were some other medium BN2 units firing in the late retraction phase as well as during the protraction phase and the interpattern interval. Our previous studies have demonstrated that these units were from B38 (McManus et al., 2014). This was consistent with the results reported by Church and Lloyd (1994), in which B38 was identified as the protraction phase motor neuron.

Block definition. Based on unit sizes (Lum et al., 2005), timing, and firing features, the BN2 motor patterns during the retraction phase (in vivo or in vitro) can be characterized by four blocks (blocks 1-4; Figs. 2A, 3A, 4A, green, yellow, red, and blue boxes). Previous studies used $3,1.5$, and $3 \mathrm{~Hz}$ frequency thresholds for detection of large, medium, and small BN2 units (Morton and Chiel, 1993a). Using these same frequencies generated results that were not significantly different from using a fixed frequency $(2 \mathrm{~Hz})$ within that range $(1.5-3 \mathrm{~Hz})$ for all BN2 units except B43, and so for this study we used $2 \mathrm{~Hz}$ to provide a uniform and simple criterion. For B43, we used $10 \mathrm{~Hz}$ for onset and $5 \mathrm{~Hz}$ for offset to detect its high-frequency bursting at the end of the retraction phase.

Block 1 contained the early activity of the third largest group of BN2 units (i.e., B10, B39, and other unidentified units, which started at around the end of I2 activity). The onset of block 1 was defined by the first spike of these small BN2 units after the end of the protraction phase (i.e., after the end of the I2 activity), whose instantaneous firing frequency (IFF) was $>2 \mathrm{~Hz}$. Because of their overlap with other small BN2 units in the later retraction phase, we could not determine the end time for these small units, so we focused on their activity before the second largest group of BN2 units. Thus we defined the end of block 1 by the first spike of the second largest group of the BN2 units whose IFF was $>2 \mathrm{~Hz}$.

Block 2 was defined by the activity of the second largest group of BN2 units (B6/B9). The onset of block 2 coincided with the end of block 1. The end of block 2 was defined by the last spike of the second largest group of the BN2 units with IFF $>2 \mathrm{~Hz}$.
A

in vivo

$\mathrm{I} 2$

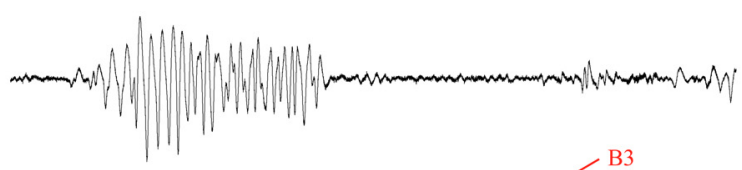

$\mathrm{BN} 2$

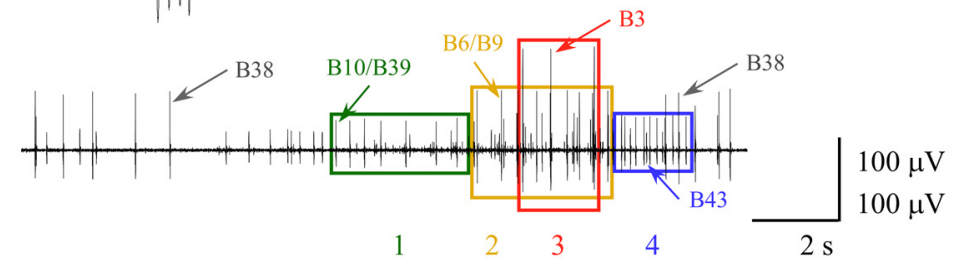

B1

suspended buccal mass
B6

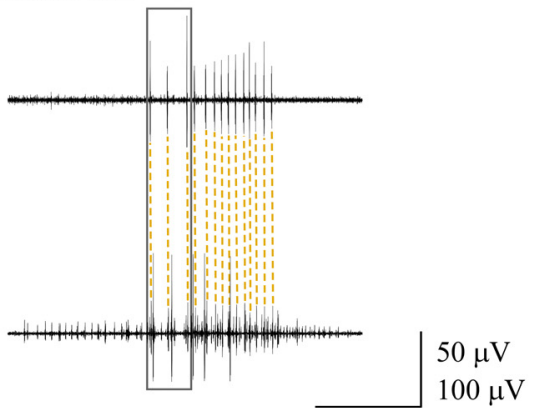

$1 \mathrm{~s}$

B2

suspended buccal mass

B9

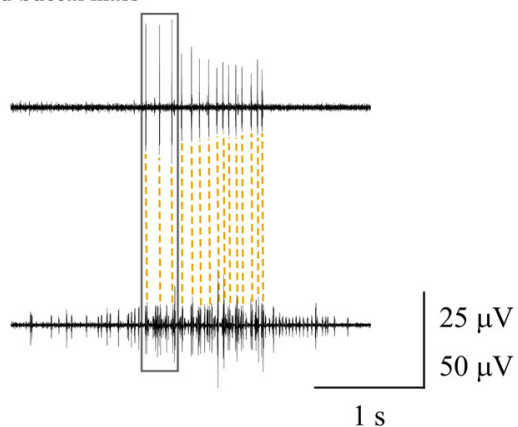

B3

suspended buccal mass

B3

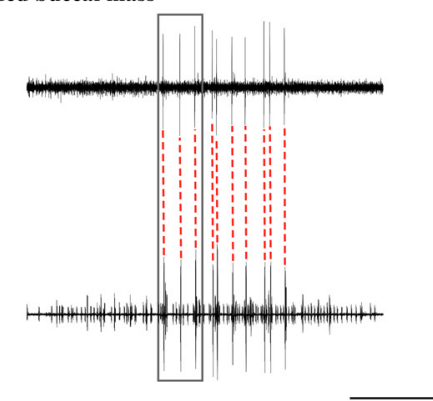

$25 \mu \mathrm{V}$

$100 \mu \mathrm{V}$

$1 \mathrm{~s}$
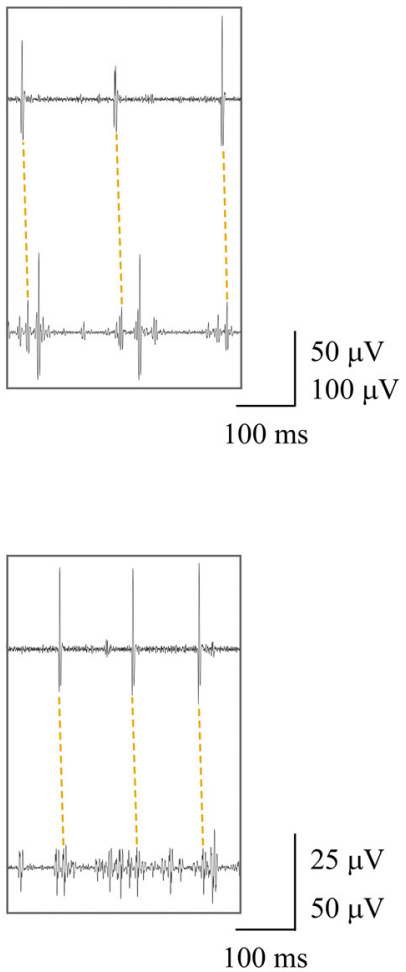

$50 \mu \mathrm{V}$

$100 \mathrm{~ms}$

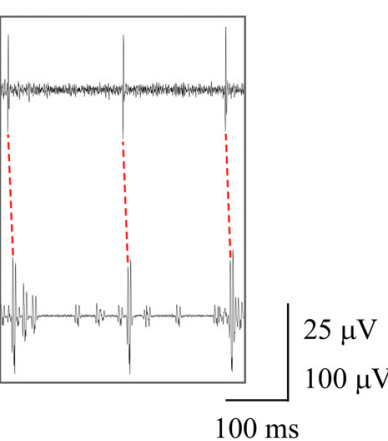

Figure 2. Characterizing the BN2 motor program patterns during the retraction phase of biting and swallowing. $\boldsymbol{A}$, The traces shown are in vivo recordings from the 12 muscle and BN2. The white bar indicates the protraction phase, defined by the activity of the 12 muscle. The black bar indicates the retraction phase, defined by the BN2 burst. The boxes in green, yellow, red, and blue correspond to blocks 1-4, respectively. B1-B3, A suspended buccal mass preparation was used for simultaneous extracellular recordings on the somata of individual motor neurons and the 2 muscle, RN, BN2, and BN3 during biting and swallowing responses (see Materials and Methods). Only the extracellular soma recordings and the BN2 recordings during the retraction phase are shown in this figure. The insets on the right side are expansions of the recordings in the gray boxes on the left side. Dashed lines (color corresponding to the block colors) indicate one-for-one correspondence between the extracellular soma spikes and units on the BN2 recording. 
A in vivo
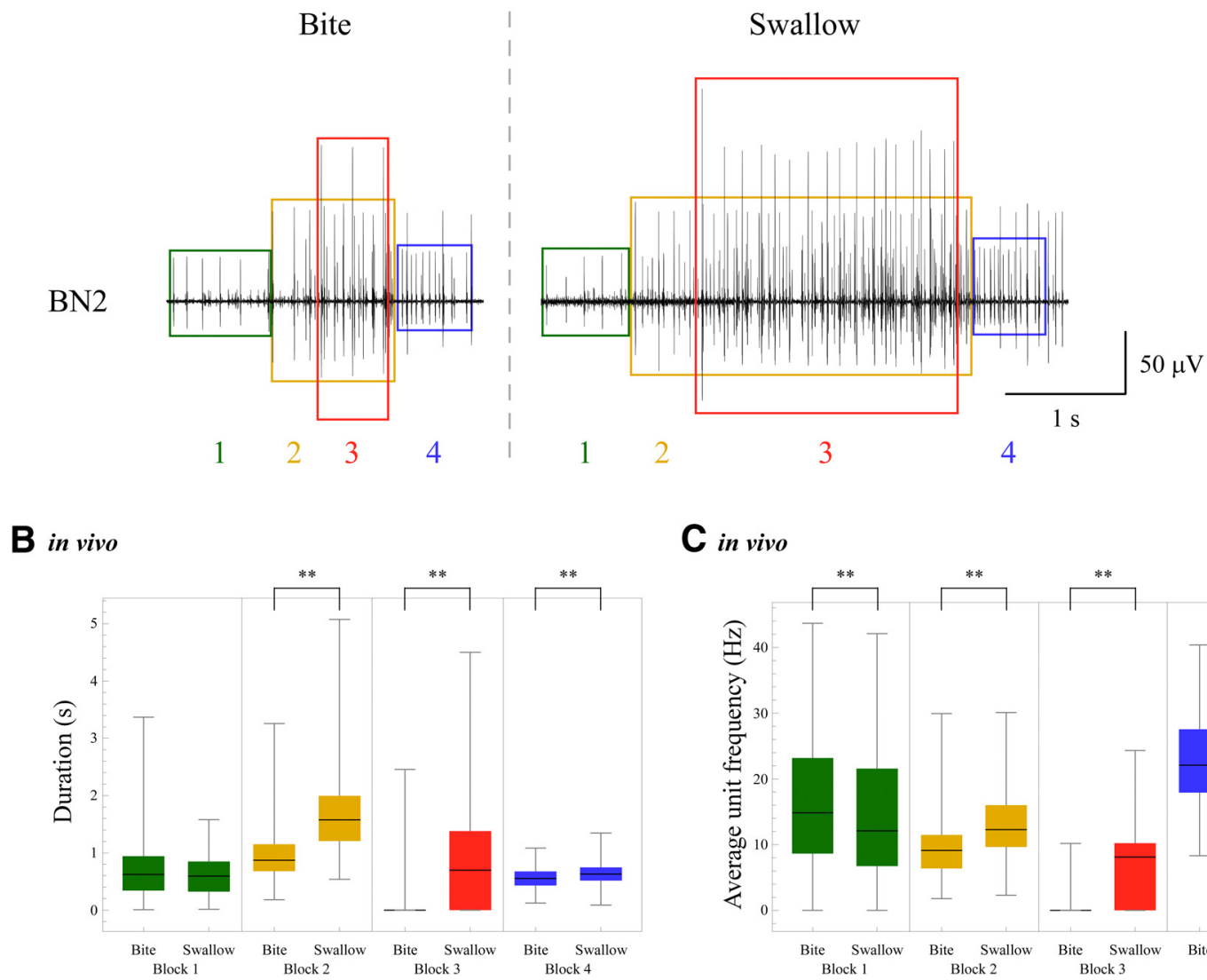

C in vivo

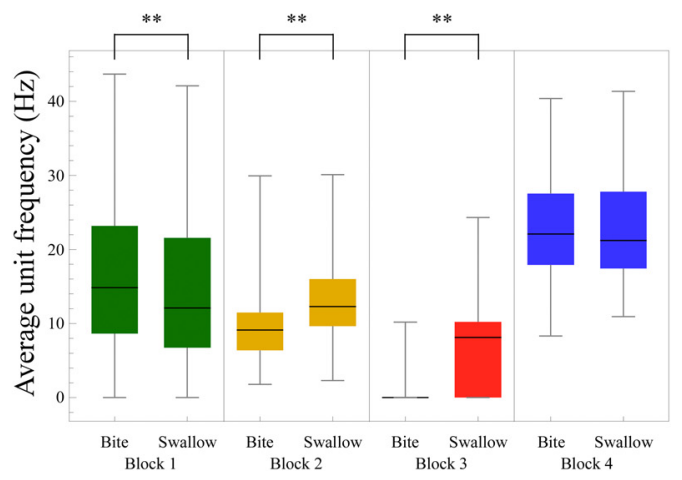

Figure 3. $B 6 / B 9$ and $B 3$ are more active in swallowing than in biting in vivo based on analyses of block duration and frequency. $A$, The $B N 2$ motor patterns during the retraction phase in biting (left) and swallowing (right) in vivo (recordings shown are from the same animal). Both types of BN2 patterns can be characterized by four blocks (Fig. 2A; see Materials and Methods). The boxes in green, yellow, red, and blue correspond to blocks 1-4, respectively. Note the longer durations and increased frequencies of blocks 2 and 3 in the swallowing pattern relative to the same blocks in the biting pattern. $\boldsymbol{B}$, The durations of blocks $2-4$ were significantly longer in swallowing than in biting, whereas the duration of block 1 was not significantly different from biting to swallowing (see Results). Because firing B43 (i.e., block 4) generates only weak forces, it is likely that only the increases in durations in blocks 2 and 3 are behaviorally significant. $C$, Average frequencies of the first (B3) and second largest units (B6/B9; i.e., units in blocks 3 and 2, respectively) were significantly higher in swallowing than in biting (see Results). In block 1, the average frequency of the third largest BN2 units was significantly lower in swallowing than in biting, so they are unlikely to contribute to force increases in swallowing. The average frequency of the third largest units in block 4 was not significantly different from biting to swallowing. Statistical summaries of the data are presented using box and whisker plots. The central line corresponds to the median value; top whisker corresponds to the maximum value, top of the box corresponds to the first quartile, bottom of the box corresponds to the third quartile, and bottom whisker corresponds to the minimum value. Two asterisks are used to indicate significance at the level of $p<0.01$ or more.

Block 3 was defined by the activity of the largest group of BN2 units (B3). The onset and end of block 3 were defined by the first and last spike of $\mathrm{B} 3$ with $\mathrm{IFF}>2 \mathrm{~Hz}$.

Block 4 contained the late activity of the third largest group of BN2 units (B43). Because the earlier activity of this neuron could not be clearly distinguished from other small units during the earlier retraction phase, we used the high-frequency bursting of this neuron at the end of the retraction phase to define block 4 . The onset of block 4 was defined by the first spike of B43 with IFF $>10 \mathrm{~Hz}$ after the end of blocks 2 and 3; the end of block 4 was defined by the last spike of B43 with IFF $>5 \mathrm{~Hz}$. Since B38 was described as the protraction phase neuron (Church and Lloyd, 1994; McManus et al., 2014; Fig. 2 A, B38 units during the protraction phase), it was excluded from block 4 even if it was firing during block 4 .

Statistical analyses. Once the data were divided into blocks, two comparisons were made for each block in biting versus swallowing (block duration and frequency of spike activity). Thus, we applied Bonferroni's correction for these comparisons [so the significance level was 0.025 $(0.05 / 2)]$. In all comparisons, the Mann-Whitney test was used because the data were not normally distributed. Similar analyses of duration and frequency were also applied to the extracellular soma recordings of B6 and $\mathrm{B} 9$ obtained in the suspended buccal mass preparation.

\section{Results}

Prior work established that biting behavior serves as a precursor for swallowing, since a bite is an attempt to grasp food that immediately transforms into a swallow once food is actually grasped (Kupfermann, 1974). To determine, at the level of individual identified motor neurons, how biting behavior might prepare the musculature for swallowing behavior, it is essential to determine which motor neurons are recruited as an animal switches from biting to swallowing, and to measure the physiological firing rates of these motor neurons in vivo. Once this is done, it becomes possible to determine how activity in the precursor behavior can affect the musculature during the subsequent behavior.

\section{The $\mathrm{I} 3$ motor neurons $\mathrm{B} 6, \mathrm{~B} 9$, and $\mathrm{B} 3$ are selectively recruited for swallowing}

To strongly pull food inwards during swallowing, motor neurons of the retraction phase are likely to be selectively activated. Previous anatomical studies suggested that the contraction of the I3 muscle mediates retraction (Howells, 1942). The I3 muscle is primarily innervated by BN2 (Scott et 


\section{A suspended buccal mass}

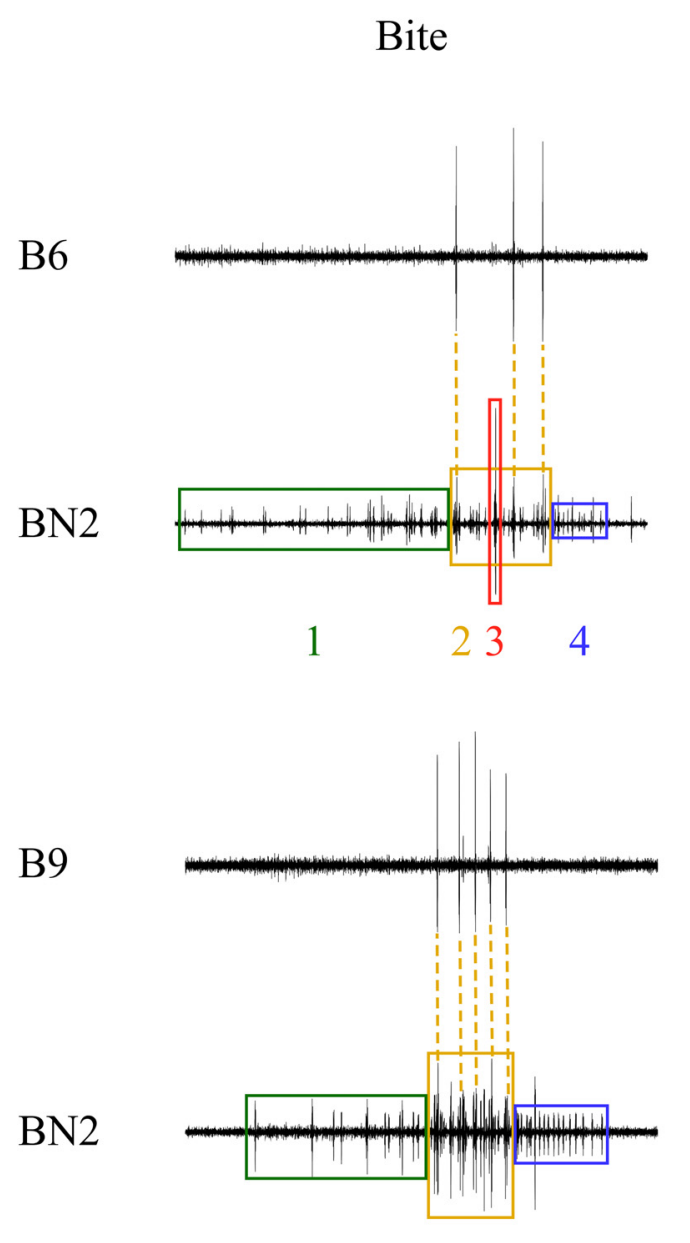

\section{B suspended buccal mass}

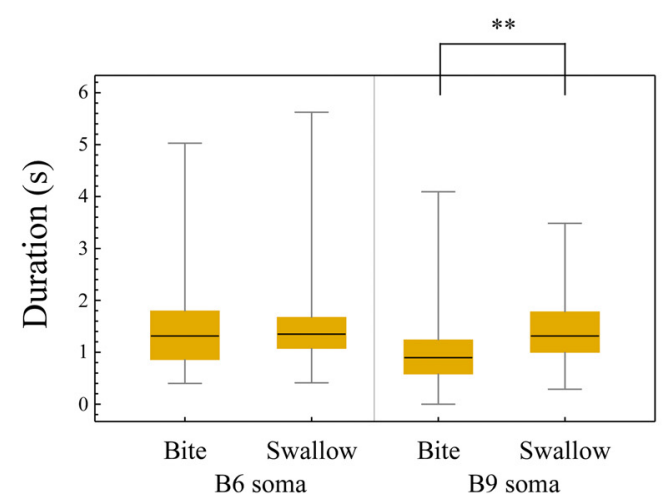

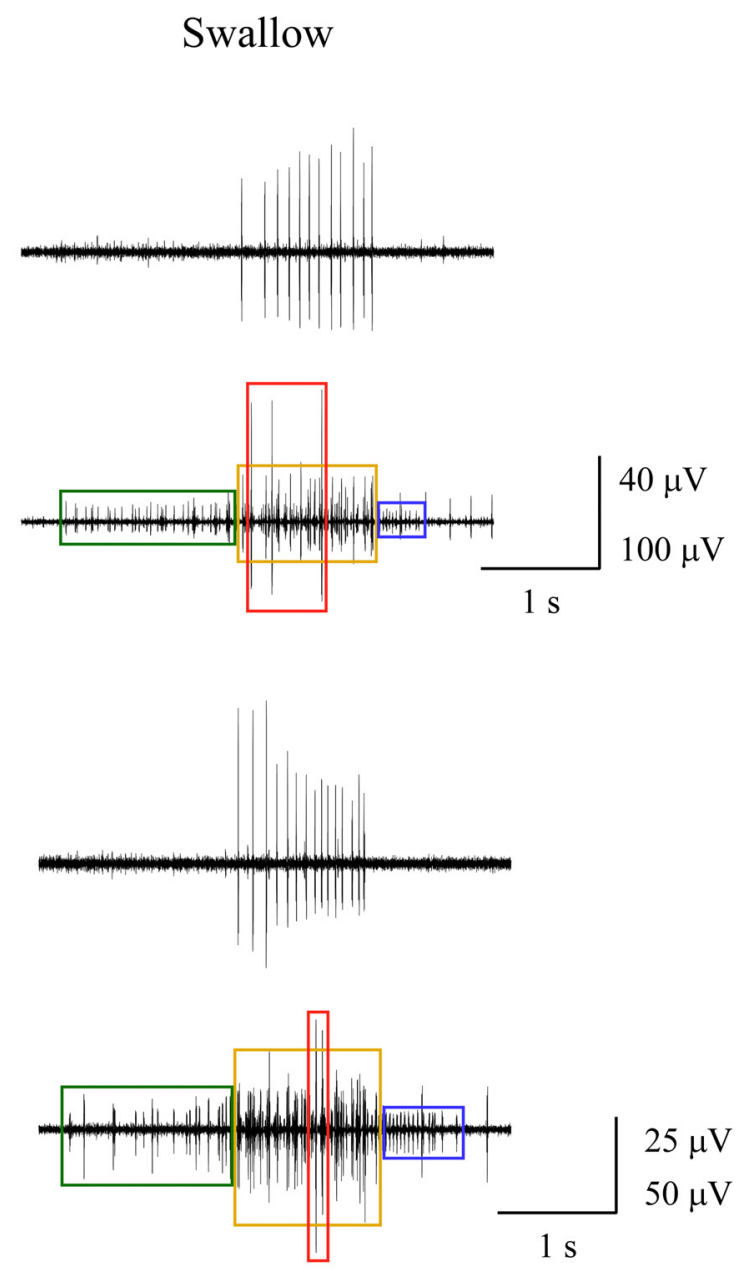

C suspended buccal mass

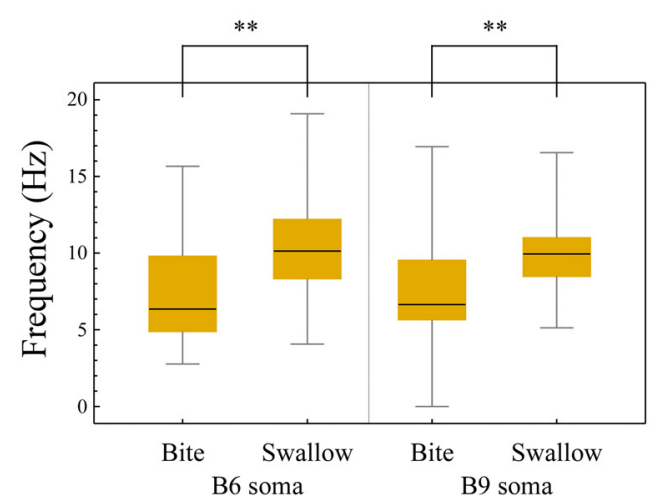

Figure 4. B6 and $B 9$ are more active in swallowing than in biting in the suspended buccal mass preparation. $A$, A suspended buccal mass preparation was used for simultaneous extracellular recordings from the somata of individual motor neurons and the 12 muscle, RN, BN2, and BN3 during biting and swallowing responses (see Materials and Methods). Only the extracellular soma recordings and the BN2 recordings during the retraction phase are shown in this figure. Green, yellow, red, and blue boxes represent blocks 1-4, respectively. Yellow dashed lines show the one-for-one correspondence of B6 and B9 soma recordings with the second largest BN2 units in block 2 in the biting response (left). Similar one-to-one correspondence is seen in the swallowing response (right), but dashed lines are not drawn for clarity. $\boldsymbol{B}$, The duration of B6 was not significantly different between biting and swallowing (see Results). However, the duration of B9 was significantly longer in swallowing than in biting (see Results). $\mathbf{C}$, The average firing frequencies of B6 and B9 were both significantly higher in swallowing than in biting (see Results). Data are presented using box and whisker plots; see Figure 3 caption for more details. Asterisks indicate significant differences at the level of $p<0.01$ or more. 
al., 1991, they referred to BN2 as nerve 5), and stimulation of $\mathrm{BN} 2$ mediates the retraction of the radula/odontophore (Morton and Chiel, 1993a; we also refer to the radula/odontophore as the grasper).

During the precursor behavior to swallowing (i.e., biting behavior), there is also a retraction phase, during which the motor neurons for the I3 muscle are also activated. How does the activity of these motor neurons change as an animal switches from biting to swallowing? To determine the protraction phase of the behaviors, we recorded from the I2 muscle (Hurwitz et al., 1996), and to monitor motor neuronal activity during retraction, we recorded from BN2 in vivo as animals generated bites or swallows (Figs. 2A, 3A; see Materials and Methods).

To determine how neural activity changes as an animal switches from biting to swallowing, we characterized the BN2 motor program patterns by examining the occurrence of activity in specific identified neurons, which led us to define four blocks of activity on BN2, based on unit size, timing, and firing features of the BN2 units (see Materials and Methods; Fig. 2A). Block 1 contains the third largest group of BN2 units (i.e., B10, B39, and other unidentified neurons) in the early retraction phase. Block 2 contains the second largest group of BN2 units (i.e., B6 and B9) in the middle retraction phase. Block 3 contains the largest group of BN2 units (i.e., B3) in the middle retraction phase. Block 4 contains the third largest group of BN2 units (i.e., B43) in the late retraction phase. We noted that the block structures were very similar from biting to swallowing (Fig. 3A), except for changes in durations and frequencies of identified motor neurons that were recruited more strongly in swallowing than in biting during blocks 2 and 3.

Motor neurons B6, B9, and B3 are very likely to be the key motor neurons mediating increased retraction forces during swallowing based on the analyses of block duration and frequency in vivo. We analyzed the block duration and frequency of the in vivo BN2 motor patterns from 162 bites and 182 swallows in five animals (Fig. 3). The average unit frequency of each block was calculated based on the number of spikes and the duration from the first spike to the last spike of the target BN2 unit group in each block. Both the durations and frequencies of blocks 2 (B6/B9) and 3 (B3) were significantly increased in swallowing compared with biting (Fig. $3 B, C$; duration medians increased from 0.87 to $1.58 \mathrm{~s}$ for block 2 , and from 0 to $0.70 \mathrm{~s}$ for block 3 ; frequency medians increased from 9.1 to $12.3 \mathrm{~Hz}$ for block 2 , and from 0 to $8.1 \mathrm{~Hz}$ for block 3; Mann-Whitney test, using Bonferroni's correction, $\alpha=0.05 / 2=0.025, p<0.0001$ for both blocks 2 and 3 in duration and frequency).

We also noted other changes in duration and frequency from biting to swallowing in vivo, but these changes were unlikely to contribute to higher forces in swallowing. For example, the duration of block 4 was longer in swallowing than in biting (representing B43 activity; Fig. $3 B ; p=0.001$ ). Since B43 generated very weak forces even when it was firing at high frequencies (e.g., 50 $\mathrm{Hz}$ ) for substantial periods of time (e.g., 2 s), it was unlikely to contribute to significant forces in swallowing, so it was not further studied. Similarly, the frequency of the third largest BN2 units in block 1 was significantly lower in swallowing than in biting (B10, B39, and other unidentified neurons; Fig. 3C; $p=$ 0.012 for block 1 ); these would therefore generate less force in swallowing, and were not further studied.

Although our studies have shown that the second block corresponds to the activity of B6 and B9 (Lu et al., 2013; Fig. 2B1,B2), it is not possible to directly establish the firing frequency of these neurons in vivo. When we attempted to identify the neurons using spike sorting, their unit shapes were too similar to distinguish one from another. Thus, we used the suspended buccal mass preparation (Fig. 1B), in which we could induce very similar block patterns in biting-like and swallowing-like movements while simultaneously recording from the somata of B6 and B9 (Figs. 2B1,B2, 4).

We applied the same duration and frequency analyses to block 2 of the suspended buccal mass data as we did to the in vivo data. The median duration of block 2 was $1.85 \mathrm{~s}$ (first quartile, $1.44 \mathrm{~s}$; third quartile, $2.28 \mathrm{~s}$; from 194 patterns in nine preparations; in comparison, in vivo median duration was $1.58 \mathrm{~s}$; first quartile, $1.21 \mathrm{~s}$; third quartile $2.0 \mathrm{~s}$ ). The median frequency was $19.0 \mathrm{~Hz}$ (first quartile, $14.2 \mathrm{~Hz}$; third quartile, $22.1 \mathrm{~Hz}$; from 194 patterns in nine preparations; in comparison, the in vivo median frequency was lower at $12.3 \mathrm{~Hz}$; first quartile, $9.65 \mathrm{~Hz}$; third quartile, $16.0 \mathrm{~Hz}$ ). Thus, the block 2 duration and frequency in the suspended buccal mass were significantly higher than in vivo (Mann-Whitney test, $\alpha=0.05 / 2=0.025, p<0.001$ ). Because of these differences, the frequencies of $\mathrm{B} 6$ and $\mathrm{B} 9$ obtained from soma recordings in the suspended buccal mass are likely to provide an upper bound on the physiological range of firing that would be observed in vivo.

Although durations were similar for B6 in biting and swallowing, the firing frequency increased in swallowing. Motor neuron B9 showed increases in both duration and frequency during swallowing. The median biting duration for B6 was $1.3 \mathrm{~s}$, whereas the median swallowing duration was $1.35 \mathrm{~s}$ (Mann-Whitney test, using Bonferroni's correction, $\alpha=0.025, p=0.85$ ). The median frequencies were $6.4 \mathrm{~Hz}$ in biting versus $10.1 \mathrm{~Hz}$ in swallowing $(p<0.0001)$. For B9, the median durations were $0.9 \mathrm{~s}$ in biting versus $1.31 \mathrm{~s}$ in swallowing $(p<0.0001)$. The median frequencies were $6.6 \mathrm{~Hz}$ in biting versus $9.9 \mathrm{~Hz}$ in swallowing $(p<0.0001)$.

\section{Activity of B6, B9, and B3 in most bites and some swallows generates minimal forces in the unmodulated $\mathrm{I} 3$ muscle}

The results thus far showed that B6, B9, and B3 might be responsible for the force difference between the retraction phases of biting and subsequent swallowing behaviors/responses. What muscle forces are evoked by these motor neurons at their physiological activity levels observed in biting and swallowing in vivo or in vitro in the suspended buccal mass preparation? As described in the Materials and Methods section, we needed to use the anchored buccal mass preparation (Fig. 1C) to measure the I3 muscle forces. Previous studies have shown that I3 can be modulated by serotonin released by the metacerebral cells (MCCs) in the cerebral ganglion (Fox and Lloyd, 1998, 2000). To suppress spontaneous neural activity of the MCCs and the I3 motor neurons during neuron stimulation, the buccal and cerebral ganglia were placed in high-divalent Aplysia saline $\left(3 \times \mathrm{Mg}^{2+}, 3 \times \mathrm{Ca}^{2+}\right.$; Morton and Chiel, 1993b) whenever motor neurons were stimulated.

We measured the forces that a neuron could generate in an unmodulated muscle when the neuron was fired over a range of durations and frequencies, up to values that generated the maximum force $(5 \mathrm{~s}, 15 \mathrm{~Hz})$. We then compared these durations and frequencies to those observed either in vivo (B3) or in the suspended buccal mass preparation (B6/B9) to see what forces would be generated by these patterns of activity. To minimize the variability in forces observed in different preparations $(n=6$ preparations for B6; $n=7$ preparations for both $\mathrm{B} 9$ and $\mathrm{B} 3$ ), the forces were normalized by the maximum force evoked by the same neuron in the same experiment, which occurred at $5 \mathrm{~s}, 15$ $\mathrm{Hz}$. Contour plots were generated based on the normalized forces for each neuron. In each plot (Fig. 5), contour lines (white or black) represent different levels of normalized forces for the spe- 
A nchored buccal mass

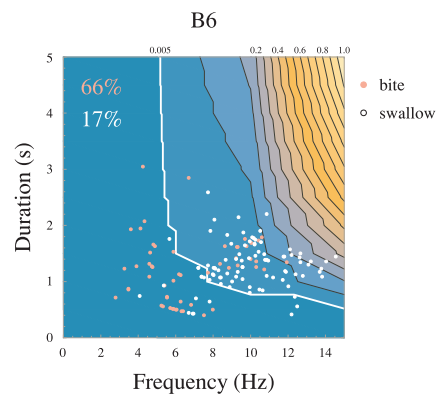

B anchored buccal mass

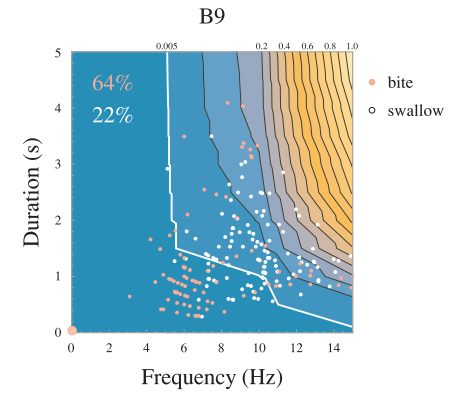

C anchored buccal mass

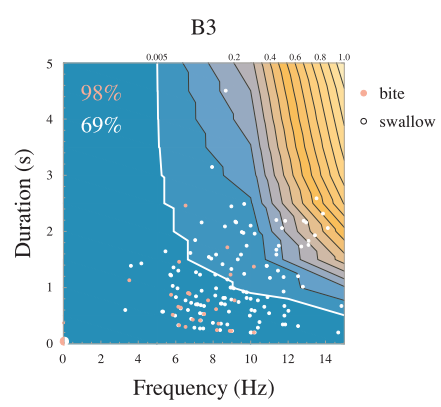

Figure 5. $B 6, B 9$, and $B 3$ generate little force in most bites and in some swallows. $A-C$, Contour plots of forces generated by individual neurons $B 6, B 9$, and $B 3$ activated at different durations (i.e., $0.5-5 \mathrm{~s}$ in increments of $0.5 \mathrm{~s}$ ) and frequencies (i.e., $5-15 \mathrm{~Hz}$ in increments of $5 \mathrm{~Hz}$ ) in the absence of neuromodulation from the MCCs by bathing the buccal and cerebral ganglia in a high-divalent cation solution $\left(3 \times \mathrm{Mg}^{2+}, 3 \times \mathrm{Ca}^{2+}\right)$. Normalized forces (see Results) were plotted against the corresponding durations and frequencies. The thick white line represents a minimal force level that is 0.005 times the maximum normalized force in each plot (see the label above each plot). Black lines represent $0.2,0.4,0.6,0.8$, and 1.0 times the maximum normalized force in each plot (see the labels above each contour region). Dots represent the duration and frequency levels of B6 or B9 neural activity (from the suspended buccal mass) or B3 neural activity (in vivo) in biting responses (pink dots) or swallowing responses (white dots). The large pink dot at $(0,0)$ in $B$ represents seven biting responses of $B 9$ obtained in the suspended buccal mass preparation. The large pink/white dot at $(0,0)$ in $C$ represents 135 bites and 46 swallows of $B 3$ obtained in vivo. Percentage values shown in $A-C$ indicate how many points fall outside the thick white line in biting (pink number) and swallowing (white number), i.e., correspond to forces $<0.005$ times the maximum possible.

cific neuron; points represent the duration and frequency levels of activation of the specific neuron in biting (pink) or swallowing (white) based on in vivo (B3: 162 bites and 182 swallows from five animals) or suspended buccal mass data (B6: 66 biting and 104 swallowing responses from five preparations; and B9: 97 biting and 121 swallowing responses from six preparations).

In the absence of the neuromodulation from the MCCs, individual neurons B6, B9, and B3 generate little force in most bites and some swallows. The majority of the neurons' duration and frequency levels in biting fell in the force regions $<0.005$ times the maximum normalized forces (Fig. 5, white lines; 66\% bites for B6, 64\% bites for B9, 98\% bites for B3). Furthermore, some of these neurons' duration and frequency levels in swallowing also fell in the force regions $<0.005$ times the maximum normalized forces (Fig. 5, white lines; 17\% swallows for B6, 22\% swallows for B9, 69\% swallows for B3). These results strongly suggest that physiological firing rates of $\mathrm{B} 6, \mathrm{~B} 9$, and $\mathrm{B} 3$ in most bites and some swallows generate minimal forces in the unmodulated I 3 muscle for behaviors.

\section{Activity of B6, B9, and B3 generates little or no force during biting-like patterns, but high forces during swallowing-like patterns in the modulated $\mathrm{I} 3$ muscle}

A possible reason for the very low forces predicted by the physiological firing rates in Figure 5 is that the neurons are acting on unmodulated muscle. If the muscle were modulated by MCCs' activity, would these patterns of activity result in large forces?

To examine forces in the modulated I 3 muscle, we measured its forces during ingestive-like motor patterns induced by application of carbachol to the anchored buccal mass preparation (Fig. 6). It has previously been shown that the MCCs in the cerebral ganglion are activated by the application of carbachol and release serotonin (Susswein et al., 1996), which modulates I3 (Fox and Lloyd, 1998, 2000). In addition, all of these carbachol-induced patterns in Figure 6 were not initial patterns, so B6, B9, and B3 have already fired repeatedly, and if they have induced any intrinsic modulation in the muscle (Fox and Lloyd, 1997; Keating and Lloyd, 1999), this would also affect the muscle response.

Patterns in vitro can be classified as biting-like or swallowinglike by using the in vivo block durations (Fig. $3 B$ ). In patterns 1 and 2, durations of blocks 2 and 3 were 0.46 and $0 \mathrm{~s}$ in pattern 1 and 1.167 and $0.006 \mathrm{~s}$ in pattern 2 (Fig. 6), all of which fell into the lower quartiles of the block durations for in vivo biting (Fig. 2B). Thus, patterns 1 and 2 were more biting-like. Pattern 3 fell between biting and swallowing. In patterns 4 and 5, the durations of blocks 2 and 3 were 2.94 and $1.11 \mathrm{~s}$ in pattern 4 and 3.58 and $2.06 \mathrm{~s}$ in pattern 5 (Fig. 6), all of which fell into or were close to the upper quartiles of the block durations for in vivo swallowing (Fig. $2 B)$. Thus, both patterns 4 and 5 were more swallowing-like.

I3 generates small or no force in biting-like patterns, even with neuromodulation from the MCCs and prior motor neuronal activity. As mentioned earlier, unlike vertebrate skeletal muscle, the action potentials of motor neurons do not cause direct action potentials in the I3 muscle, but cause EJPs. The muscle will not contract until the integrated EJPs reach a threshold (Cohen et al., 1978). Thus, it is very likely that as B6, B9, and B3 are firing at very low levels of activity in biting, the integrated EJPs are not large enough to cause force. In contrast, as is clear in patterns 4 and 5, once the threshold is exceeded by the swallowing-like physiological patterns of activity, significant forces are generated in the I3 muscle.

\section{Prior firing of B6, B9, and B3 in biting-like patterns enhances} forces generated in subsequent swallowing-like patterns

What role do the lower activity levels of motor neurons B6, B9, and $\mathrm{B} 3$ during biting play in affecting the $\mathrm{I} 3$ muscle forces? We first demonstrated that intracellularly firing B6, B9, or B3 alone at frequencies, durations, and interpattern intervals observed in vivo during biting generated no force in the $\mathrm{I} 3$ muscle (Fig. 7A, left traces). Since it is possible that combinations of the motor neurons are essential for generating forces, we intracellularly stimulated the combinations of $\mathrm{B} 6$ and $\mathrm{B} 3, \mathrm{~B} 9$ and $\mathrm{B} 3$, and $\mathrm{B} 6$ and $\mathrm{B} 9$ (Fig. 7A, right traces). These combinations also generated no force in the $\mathrm{I} 3$ muscle when the neurons were fired in biting-like patterns.

Could the pattern of motor neuronal firing during biting prepare the I3 muscle to generate larger forces in subsequent swallows? To test this, we first stimulated B6, B9, and B3 to mimic their neural activity in a swallow in the absence of any prior behaviors (Fig. 7B1, left of gray dashed lines). After a delay of $\geq 100 \mathrm{~s}$ to minimize post-tetanic potentiation and the effects of any endogenous neuromodulators released by these neurons (Fox and Lloyd, 1997, 1998; Keating and Lloyd, 1999), we stim- 


\section{A anchored buccal mass}

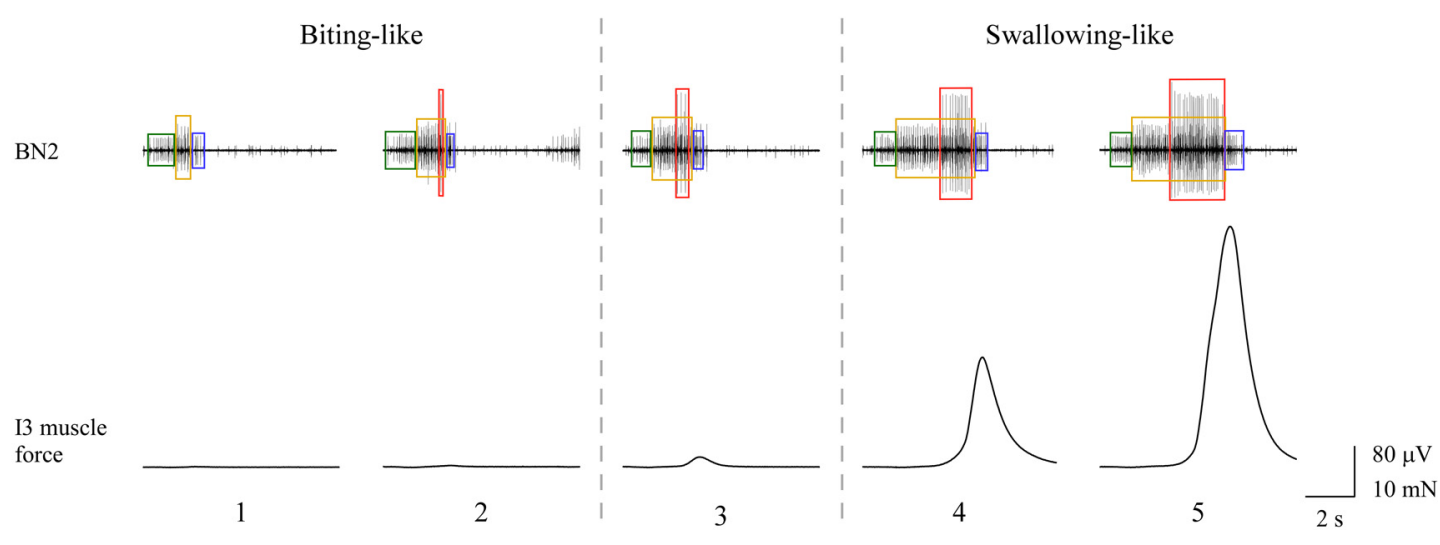

B anchored buccal mass

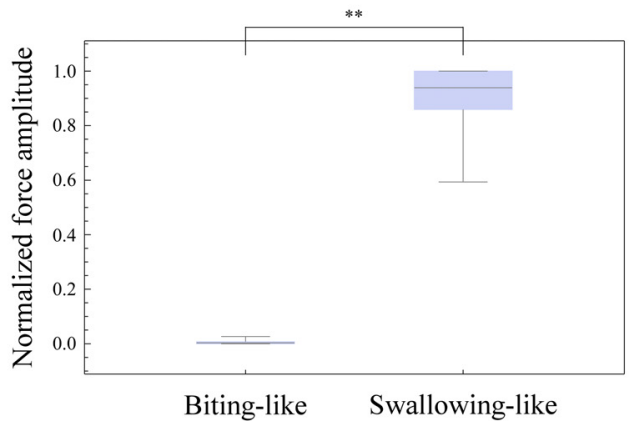

Figure 6. Biting-like patterns generate little or no force in the modulated I3 muscle, whereas swallowing-like patterns generate large forces in the modulated I3 muscle. $\boldsymbol{A}$, Five BN2 motor program patterns (top) and the corresponding force traces (bottom) during the retraction phase of ingestive-like patterns induced by application of carbachol to the anchored buccal mass preparation (see Materials and Methods and Results). The green, yellow, red, and blue boxes indicate blocks 1-4. Note that all of the patterns shown are from the same experiment and are not initial patterns, so muscles should already be modulated before each pattern after firing of the $M C C, B 6, B 9$, and B3. Using the in vivo block durations as criteria (see Results), patterns 1 and 2 were defined to be biting-like patterns; pattern 3 was intermediate between biting and swallowing; patterns 4 and 5 were swallowing-like patterns. B, Forces of biting-like and swallowing-like patterns were normalized by the maximum force in each preparation ( $n=3$ preparations, 5 biting-like and 5 swallowing-like patterns were obtained in each preparation). Biting-like patterns generate significantly smaller forces than swallowing-like patterns $\left({ }^{* *} p<0.0001\right.$, Mann-Whitney test). Data are presented as box and whisker plots.

ulated the neurons again to mimic their activity in a prior bite and the immediately following initial swallow (Fig. 7B1, right of gray dashed lines). Since B6 and B9 almost always fired in biting, we stimulated B6 and B9 with 1-10 prior biting-like patterns. Since B3 only occasionally fired in biting, we stimulated B3 with only 1-2 prior biting-like patterns.

The results were striking: even a single biting-like pattern of firing in a single motor neuron significantly enhanced the force output during a subsequent swallowing-like pattern of firing in the same motor neuron (Fig. 7B1; red dashed lines indicating the forces generated before the added biting-like pattern). The force enhancement by B6 and B9 rapidly increased as the number of the prior biting-like patterns increased from one to three and usually plateaued after five prior patterns $(n=3$ preparations for both neurons; Fig. 7B2). In contrast, the force enhancement by B3 did not change significantly after one or two prior biting-like patterns ( $n=4$ preparations; Fig. 7 B2). Therefore, although the biting-like firing of B6, B9, and B3 does not generate force during biting, it is crucial for preparing $\mathrm{I} 3$ to generate strong forces in a subsequent swallow.

Prior activity in B6, B9, and B3 either alone or in combination in swallowing-like patterns enhances forces generated by these motor neurons in subsequent swallowing-like patterns Once animals succeed in grasping a piece of seaweed, they are rarely able to ingest the entire seaweed stipe after a single swallow.
We therefore reasoned that prior swallows might also act to enhance the forces generated by subsequent swallows. Since previous studies have demonstrated that stimulation of B3 and B9 increased the contraction amplitude and relaxation rate of $\mathrm{I} 3$ evoked by the same neuron during later activation (Keating and Lloyd, 1999; Fox and Lloyd, 2001), we predicted that the firing of B6, B9, and B3 during prior swallows might enhance forces evoked by the same neuron during subsequent swallows.

We tested this hypothesis by activating B6, B9, and B3 in a sequence of swallowing-like patterns. Each neuron was repeatedly stimulated 10 times at the medians of their physiological frequency ranges of swallowing (i.e., $10 \mathrm{~Hz}$ for $\mathrm{B} 6$ and $\mathrm{B} 9$, obtained from the suspended buccal mass preparations; $8 \mathrm{~Hz}$ for B3, obtained from in vivo data) for $2 \mathrm{~s}$ (within their physiological duration ranges of swallowing) with $3 \mathrm{~s}$ interstimulus intervals to simulate their activity in swallowing (Fig. 8A). During the swallowing-like patterns, forces dramatically increased after several stimulations (from the first to the fourth stimulation) and then slowly increased until they plateaued $(n=4$ preparations for B6, $n=3$ preparations for both B9 and B3; Fig. $8 B$ ).

During both in vivo and in vitro swallowing patterns, B3 activity frequently overlaps the activity of B6 and B9, which fire simultaneously (Figs. $3 A$, right, $4 A$, right, 6, patterns 4 and 5). Could the combined activity of the different motor neurons create larger muscle forces? To test this, two different motor neurons 
A anchored buccal mass

B 6

I3 muscle

force

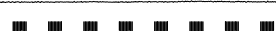

B 9

I3 muscle

force

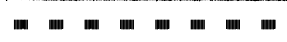

B3

I3 muscle

force
$\mathrm{B} 6$ and $\mathrm{B} 3$

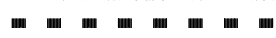

B 9 and B3

B 6 and $\mathrm{B} 9$

B1 anchored buccal mass

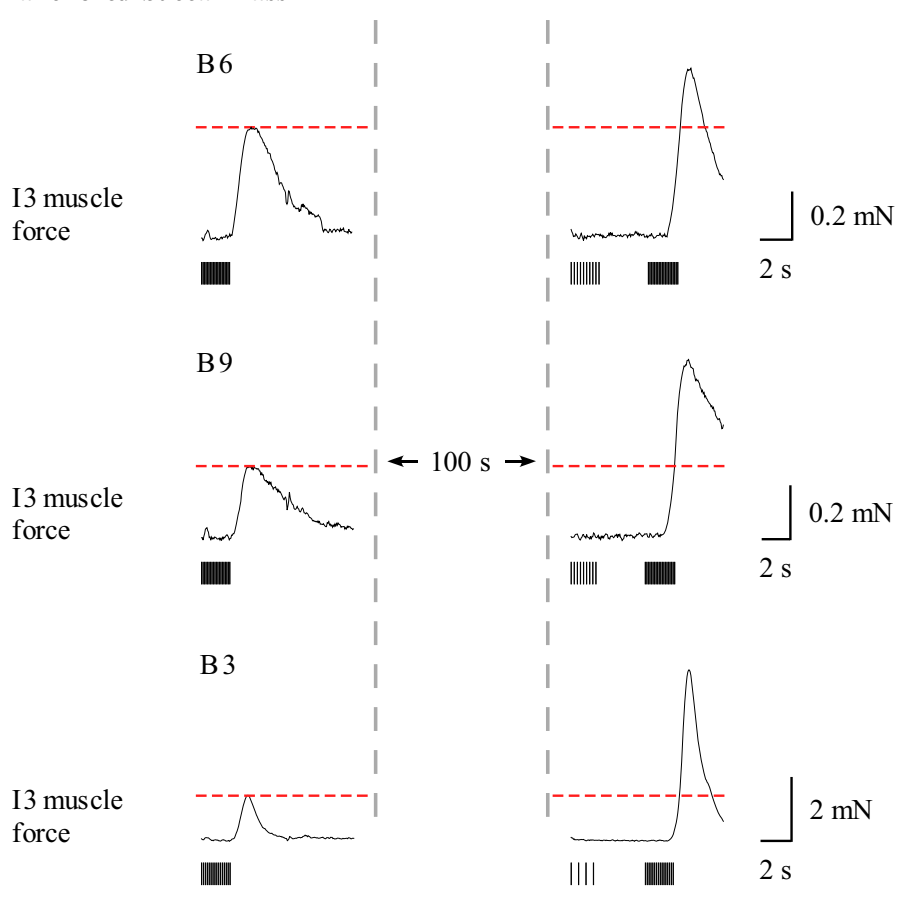

B2

anchored buccal mass
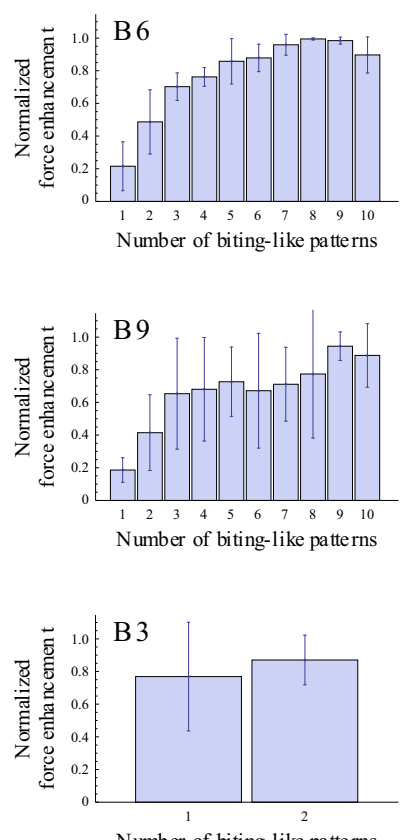

Figure 7. Activation of motor neurons $B 6, B 9$, and $B 3$ in biting-like patterns is only effective in enhancing force generation during subsequent swallowing-like patterns. $A$, Activating motor neurons B6, B9, or B3 alone or in combination at physiological durations and frequencies of biting did not generate force in the 13 muscle. Neurons were repeatedly stimulated at the median of their physiological frequency range in biting to mimic 10 sequential biting patterns either alone (left traces) or in combination (right traces). Note black bars beneath the traces indicating the stimulation protocol $(\mathrm{B} 6$ at $5 \mathrm{~Hz}, \mathrm{~B} 9$ at $5 \mathrm{~Hz}$, and $\mathrm{B} 3$ at $2 \mathrm{~Hz} ; 2 \mathrm{~s}$ duration; 3 sinterstimulus interval based on measurements of 6 sequential in vivo bites; repeated 10 times). B1, Each neuron was stimulated (black bars; $10 \mathrm{~Hz}$ for B6 and B9, $8 \mathrm{~Hz}$ for B3; $2 \mathrm{~s}$ duration) to mimic a swallowing pattern. After a 100 s rest, each neuron was first stimulated (black bars; $5 \mathrm{~Hz}$ for $\mathrm{B} 6$ and $\mathrm{B} 9,2 \mathrm{~Hz}$ for $\mathrm{B} 3 ; 2 \mathrm{~s}$ duration) to mimic a biting pattern, and then again to mimic a subsequent swallowing pattern. $\boldsymbol{B 2}$, Force enhancement for each neuron was calculated by the difference of the forces generated during the swallowing-like patterns with and without the prior biting-like patterns. It was then normalized by the maximum force enhancement that occurred in the same experiment with different numbers of the biting-like patterns. Values are reported as mean \pm SD. All differences are significant $(p<0.02$, Mann-Whitney test).

from the set of B6, B9, and B3 were stimulated both individually and together to mimic their activity in swallowing. Stimulation parameters for each neuron were the same as described above. During the swallowing-like patterns, I3 generated much larger muscle forces in response to the combined activity of two neurons than would be predicted by the sum of the forces that they generate individually (Fig. 9; $n=3$ preparations for each neuron). Therefore, both repeated firing and combined activity of multiple neurons B6, B9, and B3 can generate very large forces in swallowing. 


\section{A anchored buccal mass}

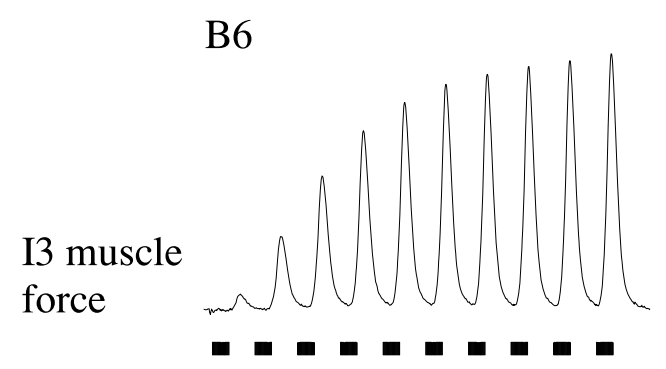

B9

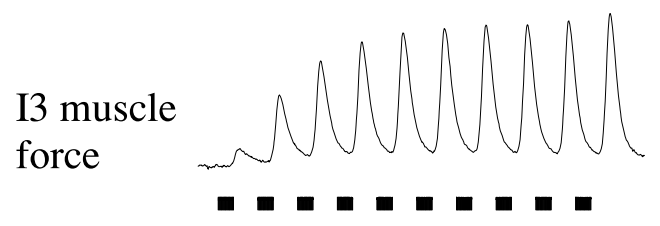

B3

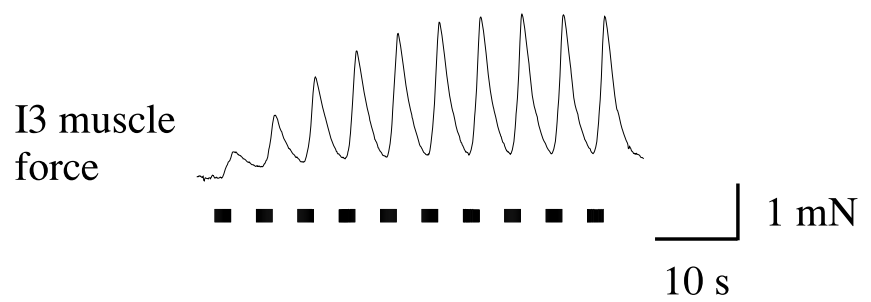

\section{B anchored buccal mass}
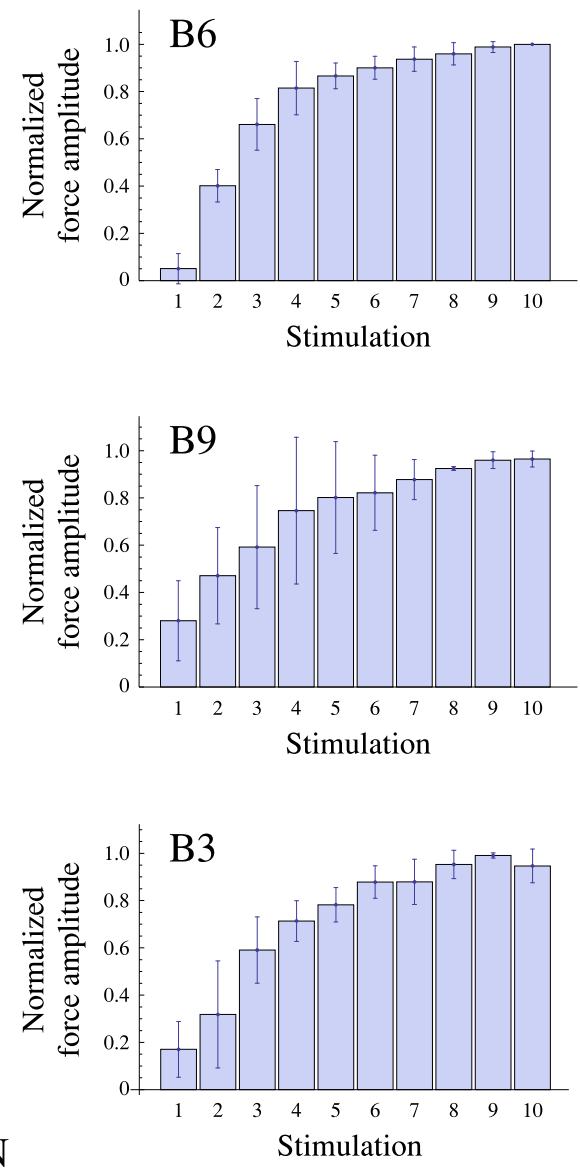

Figure 8. Repeated firing of $\mathrm{B} 6, \mathrm{~B} 9$, and $\mathrm{B} 3$ enhances the $\mathrm{I} 3$ muscle forces generated by the same neuron during swallowing-like patterns. $A$, Each neuron was repeatedly stimulated at the median of its physiological frequency range of swallowing (black bars; $\mathrm{B} 6$ at $10 \mathrm{~Hz}, \mathrm{~B} 9$ at $10 \mathrm{~Hz}$, and B3 at $8 \mathrm{~Hz} ; 2 \mathrm{~s}$ duration; 3 s interstimulus interval based on measurements from 7 sequential in vivo swallows; repeated 10 times) to mimic 10 sequential swallowing patterns. I3 generated much larger forces after repeated firing of B6, B9, and B3 during the swallowing-like patterns. $B$, Forces were normalized by the peak force evoked by the neuron during each stimulation and reported as mean $\pm \mathrm{SD}$. All differences are significant ( $p<0.02, \mathrm{Mann}-$ Whitney test).

\section{Force enhancements are not intrinsic to the $\mathrm{I} 3$ muscle and decay over many seconds}

Several decades of prior studies of the feeding musculature in Aplysia have established that peptidergic modulators released presynaptically by motor neurons can significantly modulate the forces generated due to subsequent activity of the same motor neurons (Weiss et al., 1992; Brezina, 2010; Taghert and Nitabach, 2012). The goal of the studies reported in this paper was to establish the physiological patterns of activity in motor neurons B6, B9, and B3 using in vivo and suspended buccal mass recordings, which had not been done previously, and to explore the implications of these physiological firing patterns on force generation. These results will serve as the basis for subsequent studies that can elucidate the detailed molecular mechanisms of these force enhancements.

As a first step toward this longer-term goal, we tested the hypothesis that activation of the presynaptic motor neuronal terminals is necessary for force enhancements. We compared the effects of intracellularly stimulating one of the motor neurons (B6) on muscle force development to direct muscle stimulation (Fig. 10). We observed that direct muscle stimulation (the muscle was stimulated with an extracellular hook electrode at $15 \mathrm{~Hz}$ for
$2 \mathrm{~s}$ with $3 \mathrm{~s}$ interstimulus intervals for 10 repetitions; Fig. 10B) only slightly enhanced forces during the first three stimulations, and that forces declined as stimulation continued. The averaged peak force enhancement was only $\sim 15 \%$ of the initial force $(n=$ 3 preparations). In contrast, intracellular B6 stimulation $(15 \mathrm{~Hz}$ stimulation, $2 \mathrm{~s}$ duration, $3 \mathrm{~s}$ interstimulus intervals, 10 repetitions; Fig. 10A) strongly enhanced muscle forces, which rapidly increased during the first to the fourth stimulation and then plateaued after the fifth stimulation (Fig. 10A). The averaged peak force enhancement was $\sim 210 \%$ of the initial force ( $n=3$ preparations). Thus, $>90 \%$ of the force enhancement was not intrinsic to the $\mathrm{I} 3$ muscle, suggesting that activation of the presynaptic motor neuronal terminals is necessary for these force enhancements.

Another characteristic of the modulatory effects observed in Aplysia muscles is the time course of decay, which lasts many seconds. For example, previous studies (Weiss et al., 1978) demonstrated that MCC modulation of the accessory radula closer (ARC) muscle (also known as the I5 muscle) due to serotonin release lasted 1-2 min. We therefore determined the time course of decay of the force enhancement, either due to a prior bitinglike or swallowing-like motor pattern (Fig. 11). Neurons were 


\section{A anchored buccal mass}
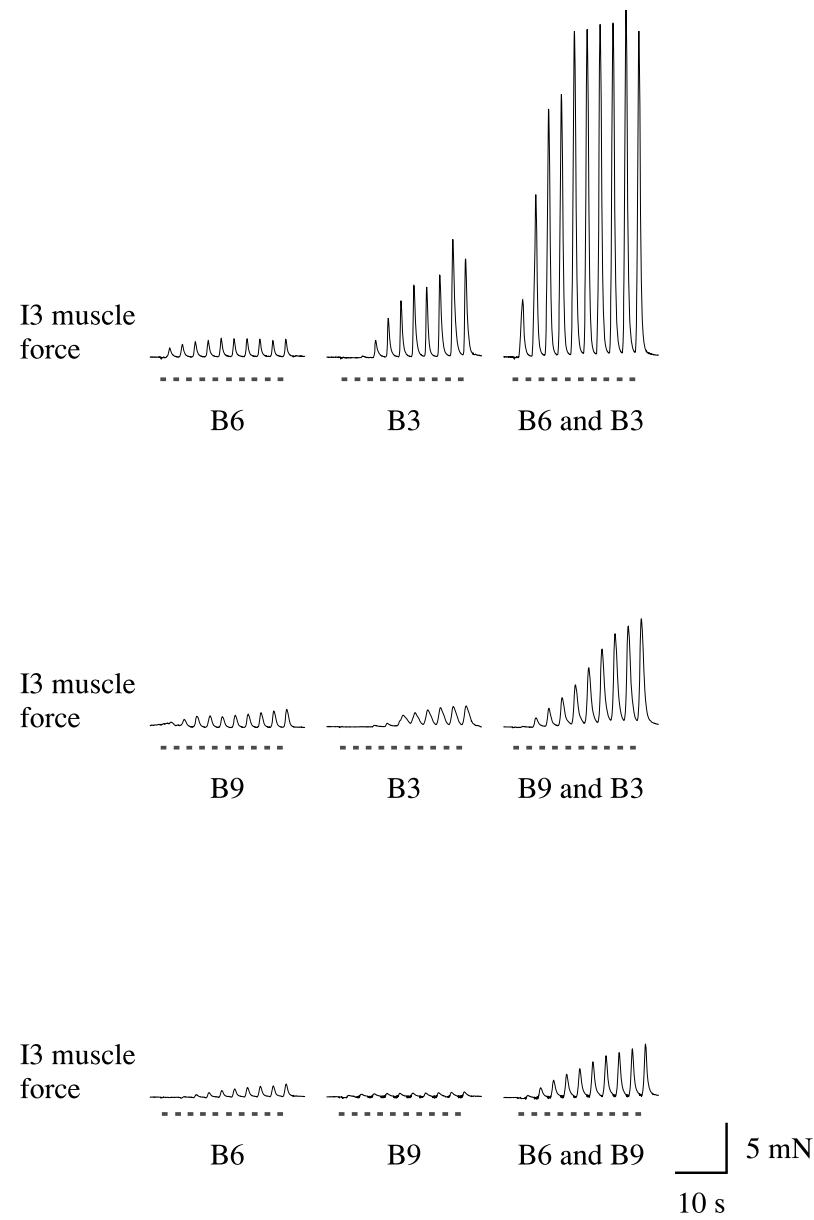

B anchored buccal mass
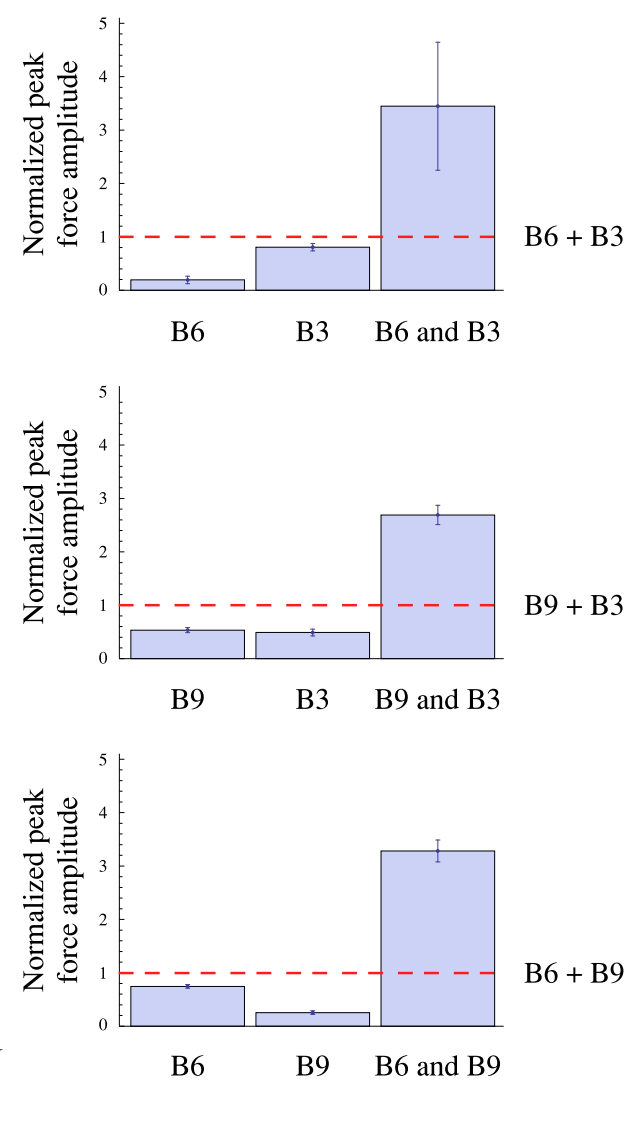

Figure 9. Combined activity of $B 6, B 9$, and $B 3$ enhances the 3 muscle forces during swallowing-like patterns. $A$, Two neurons from the set of $B 6, B 9$, and $B 3$ were stimulated both individually and simultaneously to mimic 10 continuous swallowing patterns ( $10 \mathrm{~Hz}$ for $\mathrm{B} 6$ and $\mathrm{B} 9,8 \mathrm{~Hz}$ for B3; $2 \mathrm{~s}$ duration; 3 s interstimulus interval; for 10 times). $B$, Peak forces generated by either individual or combined motor neurons in each experiment were normalized by the linear summation of the peak forces generated by each individual motor neuron (red dashed lines). Values were reported as mean $\pm S D$.

stimulated intracellularly in a biting-like or swallowing-like pattern, and then, after a variable time interval (from 0.5 to $50 \mathrm{~s}$ ), a subsequent swallowing-like pattern was induced in the same neuron, and the change in I3 force was measured. The force enhancement due to firing each of these neurons in a prior biting-like pattern returned to baseline after $25 \mathrm{~s}$ (Fig. $11 A ; n=3$ preparations for each neuron). The force enhancement due to firing B6, $\mathrm{B} 9$, or $\mathrm{B} 3$ in a prior swallowing-like pattern returned to baseline after $50 \mathrm{~s}$ (Fig. $11 B ; n=3$ preparations for both B6 and B3, $n=4$ preparations for B9). The time courses (25-50 s) of the I3 force enhancements were similar to that observed in previous studies of monoaminergic and peptiderigic modulation of these muscles (Weiss et al., 1992; Fox and Lloyd, 1998; Keating and Lloyd, 1999).

\section{Discussion}

To our knowledge, we have provided the first demonstration of a novel mechanism that prepares the musculature during an obligatory initial behavior to generate increased forces in a subsequent behavior. Specifically, we showed, first, that a major difference between biting and swallowing behavior in Aplysia is the recruitment of identified motor neurons B6, B9, and B3 (Figs. 3, 4). Then, using in vivo and suspended buccal mass recordings, we determined the physiological durations and frequencies of firing of these motor neurons, and showed that most biting and some swallowing motor patterns would not generate force in the unmodulated I 3 muscle (Fig. 5). In contrast, swallowing-like patterns do generate forces in the modulated I3 muscle, but bitinglike patterns do not (Fig. 6). The most striking result is that prior biting-like motor neuronal firing, which does not itself induce force in I3, does enhance force generation during subsequent swallowing-like motor patterns (Fig. 7). Swallowing forces are further enhanced by the activity of B6, B9, and B3 in prior swallow-like patterns, either alone or in combination (Figs. 8, 9). Finally, it is likely that the effect we have described is due to activation of the presynaptic motor neuronal terminals rather than the I3 muscle itself (Fig. 10), and the time course of the decay of muscle force enhancement due to either a prior biting-like or swallowing-like pattern is similar to the time course of decay of many previous studies of modulatory influences on muscles in the feeding apparatus of Aplysia (Fig. 11). Schematic summaries of the differential recruitment of the motor pool, and how the different patterns of neural activity affect muscle forces are provided in Figures 12 and 13, respectively.

\section{Behavioral significance}

The results presented directly support Bernstein's original hypothesis about motor coordination at the muscle level: "Co- 


\section{A anchored buccal mass}

\section{Neuron stimulation}

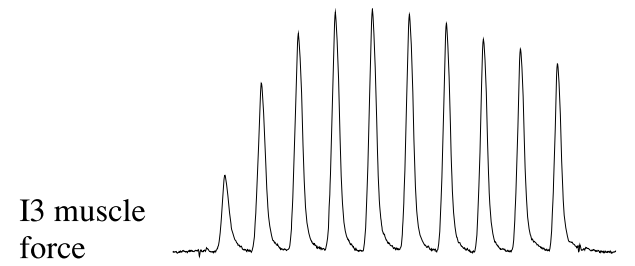

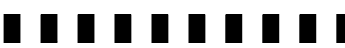

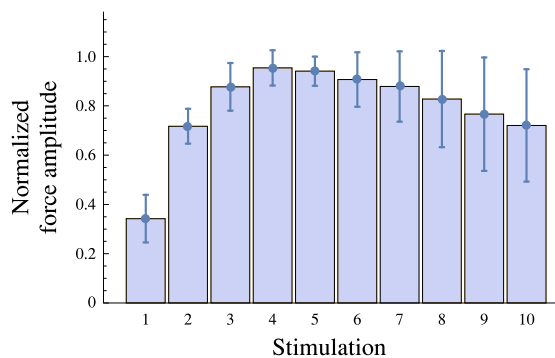

B anchored buccal mass

Muscle stimulation

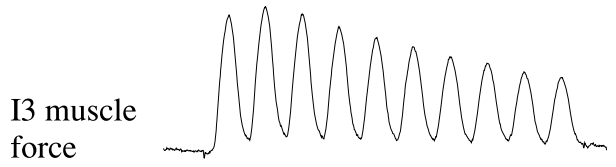

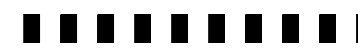
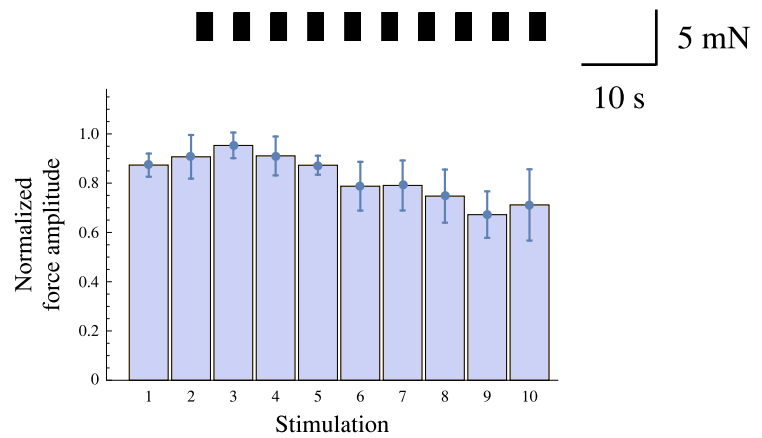

Figure 10. Comparisons between repeated neuron stimulation and muscle stimulation. $A, B 6$ was intracellularly stimulated (black bars; $15 \mathrm{~Hz} ; 2 \mathrm{~s}$ duration; $3 \mathrm{~s}$ interstimulus interval, 10 times) to mimic 10 swallowing patterns. Forces were normalized by the peak force that occurred during this stimulation and reported as mean \pm SD. $\boldsymbol{B}, 13$ was extracellularly stimulated (black bars; $15 \mathrm{~Hz}$; $2 \mathrm{~s}$ duration; 3 sinterstimulus interval, 10 times) through a hook electrode and no antidromic action potentials were induced on BN2 during the stimulation. Forces were also normalized by the peak force that occurred during this stimulation and reported as mean \pm SD. Note that during both neuron and muscle stimulations, the buccal ganglia were placed in high-divalent $A p l y s i a$ saline $(3 \times$ $\mathrm{Mg}^{2+}, 3 \times \mathrm{Ca}^{2+} ;$ Morton and Chiel, 1993b) to suppress spontaneous neural activity of the MCCs and the 13 motor neurons.

A1

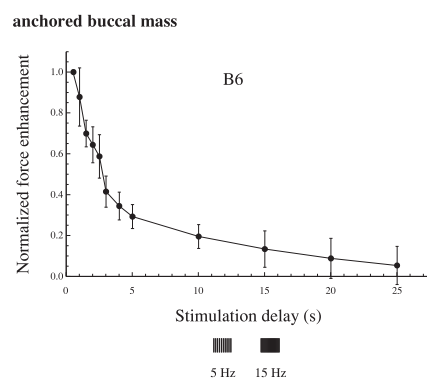

B1

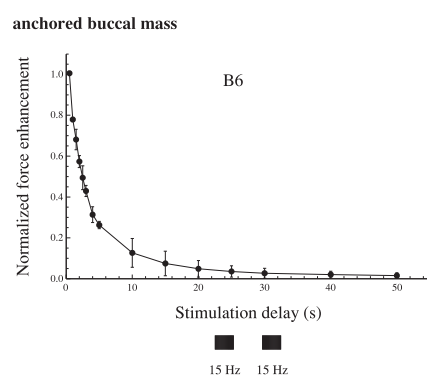

A2

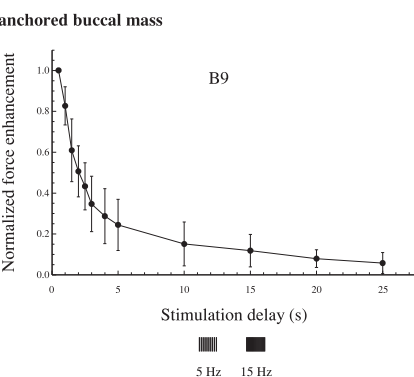

B2

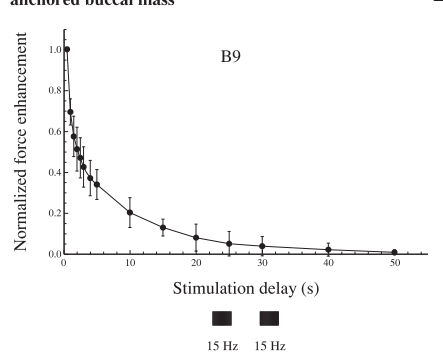

A3

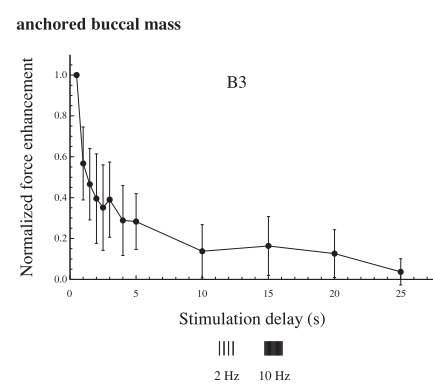

B3

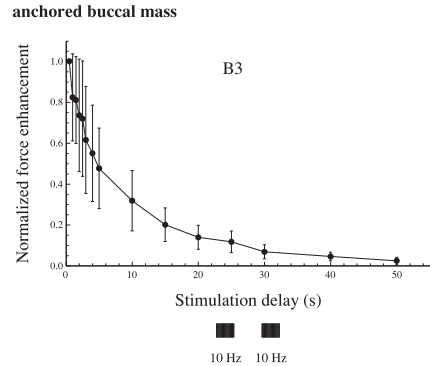

Figure 11. Force enhancement decays as the interpattern interval increases. $\mathbf{A} 1-\mathbf{A} 3$, Each neuron was first stimulated ( $5 \mathrm{~Hz}$ for $\mathrm{B} 6 \mathrm{and} \mathrm{B} 9,2 \mathrm{~Hz}$ for $\mathrm{B} 3 ; 2 \mathrm{~s}$ duration) to mimic a biting pattern. Then after a variable interval (i.e., $0.5,1,1.5,2,2.5,3,4,5,10,15,20,25 \mathrm{~s}$ ), the neuron was stimulated ( $15 \mathrm{~Hz}$ for B6 and B9, $10 \mathrm{~Hz}$ for B3; $2 \mathrm{~s}$ duration; 3 s interstimulus interval) to mimic a subsequent swallowing pattern. Force enhancement was calculated by subtracting the initial force from the second force generated during the swallowing-like pattern. Then it was normalized by the maximum force enhancement that occurred at the shortest interpattern interval (i.e., 0.5 s). Values were reported as mean \pm SD. B1-B3, Each neuron was first stimulated ( $15 \mathrm{~Hz}$ for B6 and B9, $10 \mathrm{~Hz}$ for B3; $2 \mathrm{~s}$ duration) to mimic a swallowing pattern. Then, after a variable interval (i.e., $0.5,1,1.5,2,2.5,3,4,5,10,15,20,25,30,40,50 \mathrm{~s}$ ), the neuron was stimulated again to mimic a subsequent swallowing pattern. Force enhancement values were also normalized and reported as mean \pm SD.

ordination ... lies basically not in the character and the accuracy of a tetanic effector impulse but ... of preparatory (not tetanic) effector impulses which organize and prepare the periphery for the reception of the right impulse at the right moment" (Bernstein, 1967, pp 110-111, emphasis in original). This principle is illus- trated by recent studies of motor cortical control using transcranial magnetic stimulation that suggest that just before actual grasping, the detailed motor pattern determining hand shape for grasp is transferred to primary motor cortex (Prabhu et al., 2007). In this paper, we suggest another means of preparing the periph- 
Initial Position

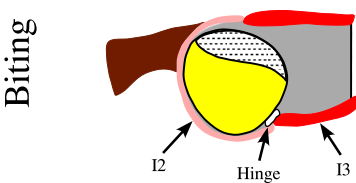

告

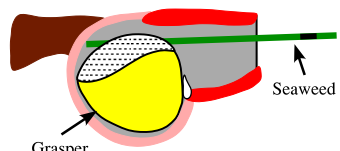

Peak Protraction

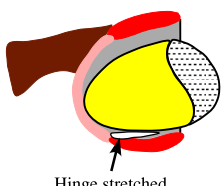

Hinge stretched

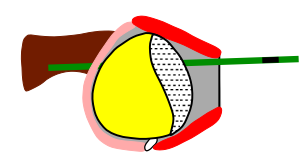

Peak Retraction
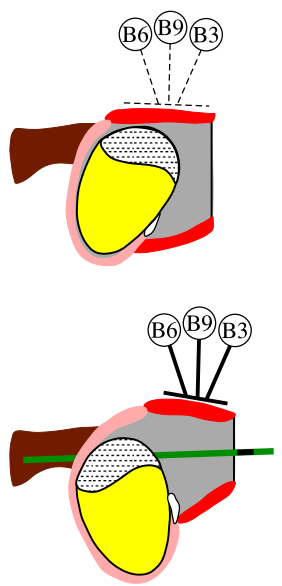

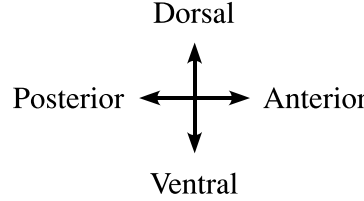

Figure 12. Schematic of motor pool function and biomechanics in biting versus swallowing. During biting, an attempt to grasp food, the grasper is strongly protracted from its resting position by the contraction of the protractor muscle ( 12 , pink), and during peak retraction, the motor neurons (B6, B9, B3) for the retractor muscle (13, red) show relatively little activation (dashed lines). Retraction is largely accomplished by contraction of the hinge (white), which is strongly stretched during biting. In contrast, during swallowing, protraction is much weaker (so as not to push food out of the mouth). The power stroke of swallowing is retraction, during which motor neurons B6, B9, and B3 are strongly activated (solid lines) and cause 3 to contract and pull seaweed (green bar; note black marker on the seaweed) inwards. Weak neural activity in biting before swallowing enhances 13 forces during swallowing, and activation of the motor neurons during prior swallows also enhances 13 forces (Fig. 13).
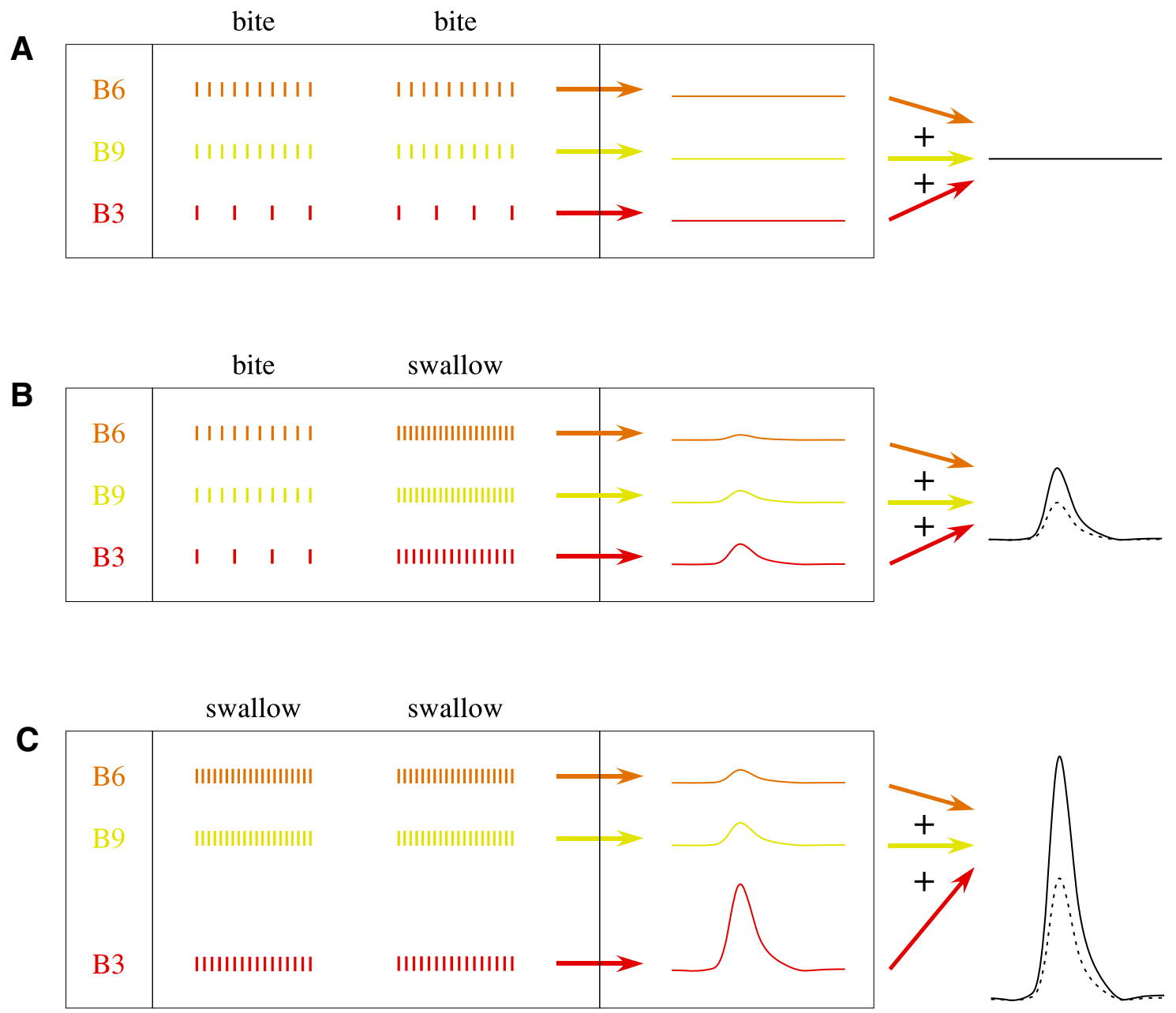

Figure 13. Summary schematics for motor neuronal activity and force generation in the 13 muscle. Orange represents the firing of B6 and the force generated by it; yellow represents the firing of $\mathrm{B} 9$ and the force generated by it; red represents the firing of $\mathrm{B} 3$ and the force generated by it. Black dashed lines on the right side represent the linear summation of forces generated by the three motor neurons; black solid lines represent the estimated nonlinear summation of forces generated by these neurons. $A, 13$ generates no muscle force in response to the firing of $B 6, B 9$, and $B 3$ in biting even with repeated stimulation of these neurons. The combined activity of multiple motor neurons does not affect the $I 3$ contraction in biting. $B$, After the firing of these neurons in prior bites, 13 generates larger forces during the initial swallow. The combined activity of $B 6, B 9$, and $B 3$ strongly enhances muscle force in the initial swallow. $C$, After the repeated firing of $B 6, B 9$, and $B 3$ in prior swallows, 13 generates much larger forces during the subsequent swallows. The combined activity of B6, B9, and B3 also strongly enhances muscle forces in subsequent swallows. 
ery: altering the ability of muscles to generate forces from one behavior to the next (Fig. 13).

Preparing I 3 for the transition from biting to swallowing is likely to be of great behavioral significance. Once animals grasp food, they must immediately clamp down on the food strongly and retract vigorously, which involves strong activation of the I3 muscle. Otherwise, animals may lose contact with food. Thus, the prior firing of $\mathrm{B} 6, \mathrm{~B} 9$, and $\mathrm{B} 3$ in biting prepares $\mathrm{I} 3$ to generate large retraction forces as soon as animals grasp food, even though it appears to play little role in biting behavior itself.

If the primary motor neurons for the retractor muscle generate little or no force during biting, how do animals retract at the end of a bite? During the strong protraction phase of biting, the "hinge," the interdigitation of the I3, I2, and I4 muscles (Sutton et al., 2004), is strongly stretched and generates sufficient force to produce the weak retractions characteristic of biting (Neustadter et al., 2007; Fig. 12). A strong retraction at the end of a bite might even be counterproductive, since it would slow the animal's ability to generate the next bite, i.e., the next attempt to grasp food.

Increasing force as animals ingest food is also behaviorally important. Once animals begin to ingest seaweed, they are rarely able to ingest the entire seaweed stipe in one swallow, and they may encounter increasing forces of resistance due to the seaweed's mechanical properties, its holdfast, or tidal surge. As a consequence, being able to generate successively stronger swallows is important, and thus the force enhancement due to the repeated firing of the I3 motor neurons may play an important role in allowing animals to generate increasingly powerful retraction forces during swallowing.

We have also observed that the force enhancement decays as the interpattern interval increases (Fig. 11). Normally, interpattern intervals are shorter in the early stages of a meal, and become longer as patterns slow down at later stages (Susswein et al., 1978; Kupfermann and Weiss, 1982). Thus, early in a meal, the repeated firing of motor neurons cause stronger force enhancement, allowing animals to perform stronger retractions that pull in more food. Once animals begin to satiate and swallowing patterns slow down, the effects of such force enhancement will be reduced, and swallows will become weaker.

How relevant are these results to vertebrate muscles? Previous studies have shown that large potentiation of muscle contraction force due to repeated stimulation is common in vertebrate fasttwitch skeletal muscles (Vandenboom et al., 2013). Our results suggest that other animals (e.g., cats, Bagust et al., 1974; rats, Abbate et al., 2000) may also use muscle activation in prior behaviors to prepare for a stronger muscle contraction in the subsequent behaviors.

\section{Mechanisms of force enhancements}

How do B6, B9, and B3 enhance forces in the I3 muscle? We have shown that the force enhancements require actions of the presynaptic motor neuronal terminals (Fig. 10), and decay with time courses similar to that shown previously for other neuromodulators in the Aplysia feeding system (Fig. 11; Weiss et al., 1978). Furthermore, previous studies have shown that $\mathrm{B} 6 / \mathrm{B} 9$ and $\mathrm{B} 3$ release conventional transmitters (B6 and B9 release acetylcholine, B3 releases glutamate), and also express and release small cardioactive peptides (SCPs) and FMRFamide ( $\mathrm{Fa}$ ) as cotransmitters, respectively (Church and Lloyd, 1991, 1994). In addition, SCPs and Fa increase the amplitude of EJPs and contractions of I3 evoked by B9 and B3, respectively (Keating and Lloyd, 1999). Thus, the force enhancements caused by the motor neurons' firing may be due to cotransmitters SCPs and Fa released by $\mathrm{B} 6 / \mathrm{B} 9$ and $\mathrm{B} 3$, respectively.

Neuromodulation due to motor neuronal activity has been referred to as intrinsic neuromodulation (Katz and Frost, 1996), and plays an important role in many different behaviors. For example, studies in Tritonia have demonstrated that the dorsal swim interneurons, which use serotonin both as a transmitter and as a modulator, have widespread modulatory effects on other neurons within the swim circuit, which are critical for normal swim behavior (Katz and Frost, 1995). Studies in the Aplysia feeding system (e.g., ARC muscle; Weiss et al., 1992) have also demonstrated that the cotransmitters released by motor neurons' (e.g., B15 and B16) firing at in vivo frequencies modify the relationship between muscle contraction amplitude and relaxation rate and thus maintain the optimal motor output when the intensity and frequency of feeding behavior change.

\section{Nonlinear summation of the combined activity of multiple motor neurons}

We have shown that the muscle forces produced by simultaneous firing of two neurons from the set of B6, B9, and B3 are much greater than the linear summation of the forces generated by each neuron separately (Fig. 9). Aplysia buccal muscles, such as I3, do not generate action potentials, but EJPs that can summate both temporarily and spatially (Cohen et al., 1978). Since the contraction amplitude is linearly correlated with the total EJP integrated over time above the threshold (Cohen et al., 1978, their Fig. 21 B), the nonlinear summation of the I3 contraction forces could be due to the greater muscle depolarization caused by nonlinear summation of EJPs (Cohen et al., 1978; McPherson and Blankenship, 1991). Nonlinear summation has also been observed in vertebrate muscles. The nonlinear summation could also be due to biomechanical reasons (e.g., cat tibialis posterior muscle, Powers and Binder, 1991) or to the geometry of the motor units in the muscle (Zuurbier and Huijing, 1992). Interestingly, studies in rat medial gastrocnemius muscle have shown nonlinear summation of multiple motor units that could be greater or less than the linear summation (Drzymała-Celichowska et al., 2010).

\section{Multifunctionality of motor neurons}

The results presented in this paper suggest that motor neurons can have multiple functions. During biting, B6, B9, and B3 may primarily act to prepare muscle for force generation so that as soon as the same neurons are recruited for swallowing, their higher frequencies and longer durations of activation can allow them to act as conventional motor neurons, rapidly generating much larger forces in the I3 retractor muscle. Prior studies also suggest that motor neurons in Aplysia are multifunctional. For example, previous work has shown that whether the B8 motor neurons act to close the grasper on material, or to both close and retract material, depends on the position of the grasper at the time the neurons are activated (Ye et al., 2006a). More generally, multifunctional peripheries are likely to flexibly use both muscles and motor neurons in multiple ways to generate related but distinct behaviors in both vertebrates and invertebrates (Gillis and Biewener, 2000; Gestreau et al., 2005; Ye et al., 2006a, b).

\section{References}

Abbate F, Sargeant AJ, Verdijk PW, de Haan A (2000) Effects of highfrequency initial pulses and post-tetanic potentiation on power output of skeletal muscle. J Appl Physiol 88:35-40. Medline

Bagust J, Lewis DM, Luck JC (1974) Post-tetanic effects in motor units of fast and slow twitch muscle of the cat. J Physiol 237:115-121. CrossRef Medline 
Bernstein N (1967) The coordination and regulation of movements. Oxford: Pergamon.

Brezina V (2010) Beyond the wiring diagram: signalling through complex neuromodulator networks. Philos Trans R Soc Lond B Biol Sci 365:2363_2374. CrossRef Medline

Burnstock G (2013) Cotransmission in the autonomic nervous system. Handb Clin Neurol 117:23-35. CrossRef Medline

Church PJ, Lloyd PE (1991) Expression of diverse neuropeptide cotransmitters by identified motor neurons in Aplysia. J Neurosci 11:618-625. Medline

Church PJ, Lloyd PE (1994) Activity of multiple identified motor neurons recorded intracellularly during evoked feedinglike motor programs in Aplysia. J Neurophysiol 72:1794-1809. Medline

Church PJ, Cohen KP, Scott ML, Kirk MD (1991) Peptidergic motoneurons in the buccal ganglia of Aplysia californica: immunocytochemical, morphological, and physiological characterizations. J Comp Physiol A 168: 323-336. Medline

Cohen JL, Weiss KR, Kupfermann I (1978) Motor control of buccal muscles in Aplysia. J Neurophysiol 41:157-180. Medline

Cullins MJ, Chiel HJ (2010) Electrode fabrication and implantation in Aplysia californica for multi-channel neural and muscular recordings in intact, freely behaving animals. J Vis Exp pii:1791. CrossRef Medline

Drzymała-Celichowska H, Krutki P, Celichowski J (2010) Summation of motor unit forces in rat medial gastrocnemius muscle. J Electromyogr Kinesiol 20:599-607. CrossRef Medline

Fox LE, Lloyd PE (1997) Serotonin and the small cardioactive peptides differentially modulate two motor neurons that innervate the same muscle fibers in Aplysia. J Neurosci 17:6064-6074. Medline

Fox LE, Lloyd PE (1998) Serotonergic neurons differentially modulate the efficacy of two motor neurons innervating the same muscle fibers in Aplysia. J Neurophysiol 80:647-655. Medline

Fox LE, Lloyd PE (2000) Role of cAMP in the short-term modulation of a neuromuscular system in aplysia. J Neurophysiol 83:1567-1579. Medline

Fox LE, Lloyd PE (2001) Evidence that post-tetanic potentiation is mediated by neuropeptide release in Aplysia. J Neurophysiol 86:2845-2855. Medline

Gallo M, Gordon T, Tyreman N, Shu Y, Putman CT (2004) Reliability of isolated isometric function measures in rat muscles composed of different fibre types. Exp Physiol 89:583-592. CrossRef Medline

Gestreau C, Dutschmann M, Obled S, Bianchi AL (2005) Activation of XII motoneurons and premotor neurons during various oropharyngeal behaviors. Respir Physiol Neurobiol 147:159-176. CrossRef Medline

Gillis GB, Biewener AA (2000) Hindlimb extensor muscle function during jumping and swimming in the toad (Bufo marinus). J Exp Biol 203:35473563. Medline

Halayko AJ, Solway J (2001) Molecular mechanisms of phenotypic plasticity in smooth muscle cells. J Appl Physiol 90:358-368. Medline

Howells HH (1942) The structure and function of the alimentary canal of Aplysia punctata. Quart J Microsc Sci 4:357-397.

Hurwitz I, Neustadter D, Morton DW, Chiel HJ, Susswein AJ (1996) Activity patterns of the B31/B32 pattern initiators innervating the I2 muscle of the buccal mass during normal feeding movements in Aplysia californica. J Neurophysiol 75:1309-1326. Medline

Katz PS, Frost WN (1995) Intrinsic neuromodulation in the Tritonia swim CPG: serotonin mediates both neuromodulation and neurotransmission by the dorsal swim interneurons. J Neurophysiol 74:2281-2294. Medline

Katz PS, Frost WN (1996) Intrinsic neuromodulation: altering neuronal circuits from within. Trends Neurosci 19:54-61. CrossRef Medline

Keating C, Lloyd PE (1999) Differential modulation of motor neurons that innervate the same muscle but use different excitatory transmitters in Aplysia. J Neurophysiol 82:1759-1767. Medline

Kupfermann I (1974) Feeding behavior in Aplysia: a simple system for the study of motivation. Behav Biol 10:1-26. CrossRef Medline

Kupfermann I, Weiss KR (1982) Activity of an identified serotonergic neuron in free moving Aplysia correlates with behavioral arousal. Brain Res 241:334-337. CrossRef Medline

Lu H, Chestek CA, Shaw KM, Chiel HJ (2008) Selective extracellular stimulation of individual neurons in ganglia. J Neural Eng 5:287-309. CrossRef Medline

Lu H, McManus JM, Chiel HJ (2013) Extracellularly identifying motor neurons for a muscle motor pool in Aplysia californica. J Vis Exp 73:e50189. CrossRef
Lum CS, Zhurov Y, Cropper EC, Weiss KR, Brezina V (2005) Variability of swallowing performance in intact, freely feeding Aplysia. J Neurophysiol 94:2427-2446. CrossRef Medline

McManus JM, Lu H, Chiel HJ (2012) An in vitro preparation for eliciting and recording feeding motor programs with physiological movements in Aplysia californica. J Vis Exp 70:e4320. CrossRef Medline

McManus JM, Lu H, Cullins MJ, Chiel HJ (2014) Differential activation of an identified motor neuron and neuromodulation provide Aplysia's retractor muscle an additional function. J Neurophysiol 112:778-791. CrossRef Medline

McPherson DR, Blankenship JE (1991) Neural control of swimming in Aplysia brasiliana. I. Innervation of parapodial muscle by pedal ganglion motorneurons. J Neurophysiol 66:1338-1351. Medline

Morton DW, Chiel HJ (1993a) In vivo buccal nerve activity that distinguishes ingestion from rejection can be used to predict behavioral transitions in Aplysia. J Comp Physiol A 172:17-32. CrossRef Medline

Morton DW, Chiel HJ (1993b) The timing of activity in motor neurons that produce radula movements distinguishes ingestion from rejection in $A p$ lysia. J Comp Physiol A 173:519-536. Medline

Neustadter DM, Herman RL, Drushel RF, Chestek DW, Chiel HJ (2007) The kinematics of multifunctionality: comparisons of biting and swallowing in Aplysia californica. J Exp Biol 210:238-260. CrossRef Medline

Powers RK, Binder MD (1991) Summation of motor unit tensions in the tibialis posterior muscle of the cat under isometric and nonisometric conditions. J Neurophysiol 66:1838-1846. Medline

Prabhu G, Voss M, Brochier T, Cattaneo L, Haggard P, Lemon R (2007) Excitability of human motor cortex inputs prior to grasp. J Physiol 581: 189-201. CrossRef Medline

Santos MJ, Kanekar N, Aruin AS (2010a) The role of anticipatory postural adjustments in compensatory control of posture: 1 . Electromyographic analysis. J Electromyogr Kinesiol 20:388-397. CrossRef Medline

Santos MJ, Kanekar N, Aruin AS (2010b) The role of anticipatory postural adjustments in compensatory control of posture: 2 . Biomechanical analysis. J Electromyogr Kinesiol 20:398-405. CrossRef Medline

Scott ML, Govind CK, Kirk MD (1991) Neuromuscular organization of the buccal system in Aplysia californica. J Comp Neurol 312:207-222. CrossRef Medline

Susswein AJ, Weiss KR, Kupfermann I (1978) The effects of food arousal on the latency of biting in Aplysia. J Comp Physiol A 123:31-41. CrossRef

Susswein AJ, Rosen SC, Gapon S, Kupfermann I (1996) Characterization of buccal motor programs elicited by a cholinergic agonist applied to the cerebral ganglion of Aplysia californica. J Comp Physiol A 179:509-524. Medline

Sutton GP, Macknin JB, Gartman SS, Sunny GP, Beer RD, Crago PE, Neustadter DM, Chiel HJ (2004) Passive hinge forces in the feeding apparatus of Aplysia aid retraction during biting but not during swallowing. J Comp Physiol A Neuroethol Sens Neural Behav Physiol 190:501-514. CrossRef Medline

Taghert PH, Nitabach MN (2012) Peptide neuromodulation in invertebrate model systems. Neuron 76:82-97. CrossRef Medline

Vandenboom R, Gittings W, Smith IC, Grange RW, Stull JT (2013) Myosin phosphorylation and force potentiation in skeletal muscle: evidence from animal models. J Muscle Res Cell Motil 34:317-332. CrossRef Medline

Warman EN, Chiel HJ (1995) A new technique for chronic single-unit extracellular recording in freely behaving animals using pipette electrodes. J Neurosci Methods 57:161-169. CrossRef Medline

Weiss KR, Cohen JL, Kupfermann I (1978) Modulatory control of buccal musculature by a serotonergic neuron (metacerebral cell) in Aplysia. J Neurophysiol 41:181-203. Medline

Weiss KR, Brezina V, Cropper EC, Hooper SL, Miller MW, Probst WC, Vilim FS, Kupfermann I (1992) Peptidergic co-transmission in Aplysia: functional implications for rhythmic behaviors. Experientia 48:456-463. CrossRef Medline

Ye H, Morton DW, Chiel HJ (2006a) Neuromechanics of coordination during swallowing in Aplysia californica. J Neurosci 26:1470-1485. CrossRef Medline

Ye H, Morton DW, Chiel HJ (2006b) Neuromechanics of multifunctionality during rejection in Aplysia californica. J Neurosci 26:10743-10755. CrossRef Medline

Zuurbier CJ, Huijing PA (1992) Influence of muscle geometry on shortening speed of fibre, aponeurosis and muscle. J Biomech 25:1017-1026. CrossRef Medline 